\title{
Impaired Glymphatic Transport in Spontaneously Hypertensive Rats
}

\author{
-Kristian Nygaard Mortensen, ${ }^{1}$ Simon Sanggaard, ${ }^{1,2}{ }^{-}$Humberto Mestre, ${ }^{3}$ Hedok Lee, ${ }^{2}$ Serhii Kostrikov, ${ }^{1}$ \\ (D)Anna L.R. Xavier, ${ }^{1}$ (D)Albert Gjedde, ${ }^{4,5}$ CHelene Benveniste, ${ }^{2}$ and Maiken Nedergaard ${ }^{1,3}$ \\ ${ }^{1}$ Center for Translational Neuromedicine, University of Copenhagen, Copenhagen N, Denmark, 2200, 2Department of Anesthesiology, Yale School of \\ Medicine, Yale University, New Haven, Connecticut 06520-8051, ${ }^{3}$ Center for Translational Neuromedicine, University of Rochester Medical Center, \\ Rochester, New York 14642, ${ }^{4}$ Departments of Clinical Research and Nuclear Medicine, Odense University Hospital, University of Southern Denmark, \\ Odense C, Denmark 5000, and 5Department of Neurology and Neurosurgery, Montreal Neurological Institute, McGill University, Montreal, Quebec H3A \\ 2B4, Canada
}

The glymphatic system is a brainwide CSF transport system that uses the perivascular space for fast inflow of CSF. Arterial pulsations are a major driver of glymphatic CSF inflow, and hypertension that causes vascular pathologies, such as arterial stiffening and perivascular alterations, may impede the inflow. We used dynamic contrast-enhanced MRI to assess the effect of hypertension on glymphatic transport kinetics in male young and adult spontaneously hypertensive (SHR) rats compared with age-matched normotensive Wistar-Kyoto rats (WKY). We anesthetized the rats with dexmedetomidine/isoflurane and infused paramagnetic contrast (Gd-DOTA) into the cisterna magna during dynamic contrast-enhanced MRI to quantify glymphatic transport kinetics. Structural MRI analysis showed that cerebroventricular volumes are larger and brain volumes significantly smaller in SHR compared with WKY rats, regardless of age. We observed ventricular reflux of Gd-DOTA in SHR rats only, indicating abnormal CSF flow dynamics secondary to innate hydrocephalus. One-tissue compartment analysis revealed impeded glymphatic transport of Gd-DOTA in SHR compared with WKY rats in both age groups, implying that glymphatic transport, including solute clearance from brain parenchyma, is impaired during evolving hypertension in young SHR, an effect that worsens in states of chronic hypertension. The study demonstrates the suppression of glymphatic clearance in SHR rats and thus offers new insight into the coexistence of hypertension and concomitant vascular pathologies in Alzheimer's disease. The study further highlights the importance of considering the distribution of tracers in the CSF compartment in the analysis of the glymphatic system.

Key words: contrast agent; CSF transport kinetics; middle cerebral artery; perivascular spaces; small vessel disease; volumetric MR

\section{Significance Statement}

The glymphatic system contributes to the removal of amyloid $\beta$ from the brain and is disrupted in Alzheimer's disease and aging. Using a rat model of hypertension, we measured gross CSF flow and tracked glymphatic influx and efflux rates with dynamic contrast-enhanced MRI, showing that glymphatic transport is compromised in both early and advanced stages of hypertension. The study provides a new perspective on the importance for brain metabolite and fluid homeostasis of maintaining healthy blood vessels, an increasingly pertinent issue in an aging population that in part may explain the link between vascular pathology and Alzheimer's disease.

\section{Introduction}

While Alzheimer's disease $(\mathrm{AD})$ is the most common cause of dementia, at least 15 million people worldwide currently suffer

Received July 31, 2018; revised March 31, 2019; accepted May 30, 2019.

Author contributions: K.N.M., S.S., H.L., S.K., A.L.R.X., and H.B. performed research; K.N.M., S.S., H.M., H.L., A.L.R.X., A.G., H.B., and M.N. analyzed data; K.N.M. and M.N. edited the paper; K.N.M., S.S., H.M., A.L.R.X., A.G., H.B., and M.N. wrote the paper; H.L., A.L.R.X., H.B., and M.N. designed research.

This work was supported by Lundbeck (R155-2016-552 and R249-2017-1511) and Novo Nordisk Foundations, the Olav Thorn Foundation, National Institutes of Health (1R01NS100366), Fondation Leducq (16CVD05), and Horizon 2020 (666881, SVD@target). from dementia arising wholly or in part from small vessel disease (SVD) of the brain. Vascular pathologies, including hypertension, are risk factors for AD (Hachinski, 2015). SVD is common in patients with chronic hypertension, and can progress silently

The authors declare no competing financial interests.

Correspondence should be addressed to Maiken Nedergaard at nedergaard@sund.ku.dk.

S. Kostrikov's present address: Department of Health Technology, Technical University of Denmark, Lyngby, Denmark, 2800.

https://doi.org/10.1523/JNEUROSCl.1974-18.2019

Copyright $\odot 2019$ the authors 


\section{a Glymphatic influx routes}
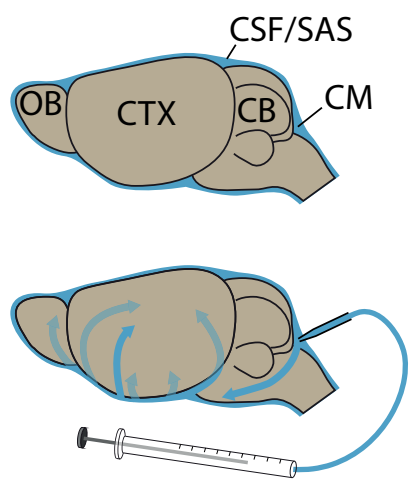

d Simplified compartments

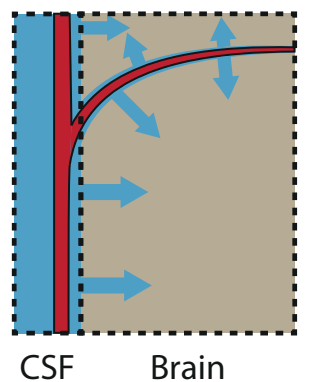

e

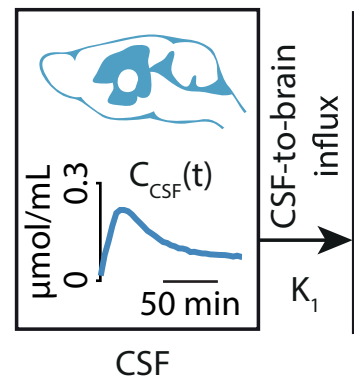

Healthy glymphatic CSF/ISF-exchange

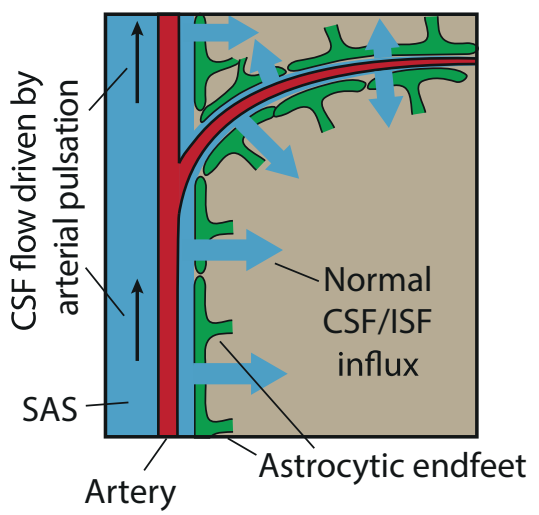

C

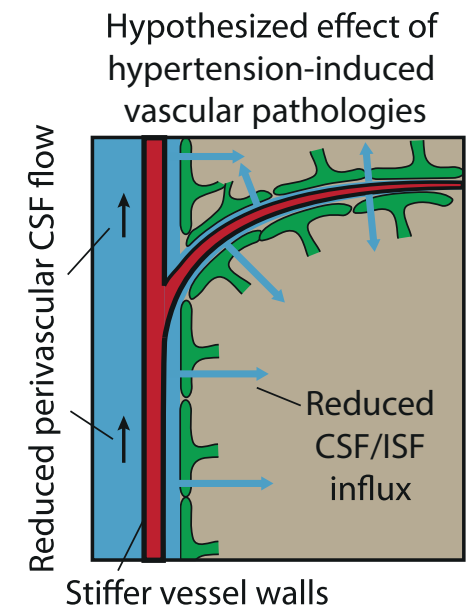

Figure 1. $\quad \boldsymbol{a}$, Overview of tracer transport in subarachnoid (SF and PVSs. $\boldsymbol{b}$, In the healthy brain, arterial pulse waves propagate the perivascular (SF via the subarachnoid space (SAS), driving a convective CSF influx into the brain, where it exchanges with the ISF to drive the glymphatic system, here shown in a simplified model. $c$, We hypothesized that hypertension-induced vascular pathology, including stiffening and reduced elasticity of the vessel wall, diminishes the ability of the arterial pulse waves to propagate the perivascular CSF, thus reducing influx of CSF into the brain in hypertensive rats. $\boldsymbol{d}$, Here, the complex intracranial space of CSF, PVSs, brain parenchyma, Virchow-Robin spaces, ISF, and vasculature was segmented into two simplified compartments: CSF and brain based on MR images. In these two compartments, TCCs were measured as the average Gd-DOTA concentration over time. e, A one-tissue compartment was fitted to the TCCs of the two compartments using the CSF TCC as the input function and the brain TCC as the tissue function. From this, we calculated the glymphatic influx rate $K_{1}$ and efflux rate constant $k_{2}$. $\boldsymbol{f}$, In the case of whole-brain analysis, partial volume effects and imperfect segmentation of the intracranial space can lead to inclusion of CSF in the brain compartment. This inclusion is modeled as the volume of CSF $\left(V_{\text {CSF }}\right)$, analogous to the blood volume in classical kinetic analysis.

for years before becoming clinically evident as cognitive dysfunction or dementia (Wardlaw et al., 2013). The most common kind of SVD is linked to arteriolosclerosis (i.e., to stiffening and reduced elasticity of small arteries and arterioles), and most commonly associated with aging, smoking, diabetes, and hypertension (Pantoni, 2010). In chronic hypertension, the increased strain on arteries remodels the arterial wall (i.e., smooth muscle hypertrophy, reduction of the lumen-media ratio, increase of collagen content, and fatigue and fracture of elastin lamellae) (Iadecola and Davisson, 2008; O'Rourke et al., 2010; Mitchell, 2014).

The glymphatic system is a brainwide fluid transport system that uses the periarterial space for fast convective inflow of CSF, driven by arterial pulsation (Iliff et al., 2012, 2013b; Mestre et al., 2018a). Fluid exchange between CSF and interstitial fluid (ISF) is facilitated by aquaporin-4 (AQP4) water channels, expressed at high density on astrocytic endfeet membranes that form the outer wall of the perivascular space (PVS) (Iliff et al., 2012; Mestre et al., 2018a,b). ISF and waste solutes drain along the perivenous space of deep central veins and venous sinuses before exiting the brain parenchyma via authentic lymphatic vessels located at the level of the dura covering the brain and cranial nerves (Aspelund et al., 2015; Louveau et al., 2015), which ultimately connects with cervical lymphatic vessels (Holland et al., 2015; Mathieu et al., 2017).
Recently, acute hypertension induced by angiotensin II infusion was shown to alter vessel wall pulsations, increasing backflow and thereby reducing net flow of CSF in the PVS (Mestre et al., 2018a).

Given the pulsation of arteries that drives the CSF-ISF exchange (Iliff et al., 2013b; Mestre et al., 2018a), we hypothesized that effects of chronic hypertension, including stiffening and reduced elasticity of the vessel wall (Iadecola and Davisson, 2008), may reduce the effectiveness of the arterial pulsation as a driver of CSF-ISF and thus impair glymphatic transport (Fig. 1). To test the hypothesis that CSF-ISF exchange is reduced with prolonged hypertension, we used quantitative dynamic contrast-enhanced MRI (DCE-MRI) (Lee et al., 2018) in combination with infusion of Gd-DOTA, into the cisterna magna (CM) to measure glymphatic transport in spontaneously hypertensive rats (SHR), an established model of the effect of hypertension on microvessels in brain (Kaiser et al., 2014), and normotensive control (WistarKyoto) rats. Two age groups were included: one representing the early emergence of hypertension (7-9 weeks) and the other the established phase of chronic hypertension (19-21 weeks). We quantified the rate of whole-brain glymphatic CSF-ISF exchange by applying a single tissue-compartment model to CSF and brain Gd-DOTA time concentration curves (TCCs). To validate that the whole-brain compartmental analysis was not biased by avail- 
ability of tracer to the glymphatic system due to differences in gross CSF flow caused by hydrocephalus, a condition inherent in SHR rats (Tajima et al., 1993; Martínez-Peña y Valenzuela et al., 2006), we also determined the local parenchymal influx rate from the PVS of the middle cerebral artery (MCA) to the proximal brain tissue.

The study identified differences in brain anatomy and CSF flow dynamics of young SHR compared with WKY that were exacerbated in the adult SHRs. Supporting the main hypothesis, glymphatic influx was significantly lower in young and adult SHR, and glymphatic efflux, or clearance, was reduced in adult SHR compared with normotensive controls. Based on these novel data, we propose that untreated hypertension may impair glymphatic system function, thereby contributing to accelerated brain aging in hypertensive patients.

\section{Materials and Methods}

\section{Compartmental modeling of Gd-DOTA transfer from CSF to brain}

We tested the hypothesis that the glymphatic CSF-ISF exchange is limited in hypertension. To this end, we compared hypertensive and normotensive rats by quantitative DCE-MRI to derive serial 3D maps of the concentration of gadolinium during and after the infusion of gadoteric acid (Gd-DOTA) into the CSF of the CM (see below). We then kinetically modeled the rate of tracer solution influx into brain tissue from CSF and the efflux from the brain of the tracer solution.

Tracer mixes with CSF after infusion into the CM, and the tracer solution undergoes transport into the subarachnoid CSF along the PVS surrounding major arteries, predominantly in the direction of arterial blood flow (Fig. 1a), entering the Virchow-Robin spaces of the penetrating arterioles. From here, AQP4 channels in the membranes of astrocytic vascular endfeet facilitate the passage of CSF tagged with the tracer into ISF (Fig. 1b). As arterial pulsation is a driver of this CSF-ISF exchange (Iliff et al., 2013b; Mestre et al., 2018a), and since arterial distensibility is reduced in chronic hypertension (Baumbach and Heistad, 1988), we hypothesize that the CSF-ISF exchange is reduced in an animal model of essential hypertension, SHR.

Here, we modeled the tracer solution transport system by two compartments: (1) an input compartment consisting of CSF; and (2) a tissue compartment consisting of brain tissue (Fig. 1d). According to this model, the tracer solution is transported from the CSF compartment to brain with the influx coefficient $K_{1}$ quantified as a clearance with unit of milliliters of tracer solution per cubed centimeter brain tissue per minute $\left(\mathrm{ml} \cdot \mathrm{cm}^{-3} \cdot \min ^{-1}\right)$ and subsequently leaves the brain tissue with the efflux rate constant $k_{2}\left(\mathrm{~min}^{-1}\right)$ (Fig. 1e,f). For the tissue compartment, we applied the following simple model to calculate $K_{1}$ and $k_{2}$ (Phan et al., 2017), as follows:

$$
\frac{d M_{\mathrm{B}}(t)}{d t}=K_{1} C_{\mathrm{CSF}}(t)-k_{2} M_{\mathrm{B}}(t)
$$

with the parameters $K_{1}$ and $k_{2}$, where $M_{\mathrm{B}}(t)$ is the average Gd-DOTA mass per tissue volume $\left(\mu \mathrm{mol} / \mathrm{cm}^{3}\right)$ at time $t$, and $C_{\mathrm{CSF}}(t)$ is the average CSF Gd-DOTA concentration $(\mu \mathrm{mol} / \mathrm{ml})$ at time $t$. The estimates of $K_{1}$ and $k_{2}$ yielded the tissue volume of distribution of the tracer as follows:

$$
V_{\mathrm{ECS}}=\frac{K_{1}}{k_{2}},
$$

where $V_{\mathrm{ECS}}$ is an estimate of an apparent volume of tracer solvent in a quantity of brain tissue, with the unit of $\mathrm{ml} / \mathrm{cm}^{3}$ and a magnitude close to the volume of the ISF, because Gd-DOTA as an extracellular tracer only distributes in the ISF of the brain.

We then applied an extension of this model to the whole brain with an additional compartment in the shape of the tracer input compartment of the entire CSF, modeled with a third parameter of the CSF volume $\left(V_{\mathrm{CSF}}\right)$. The CSF volume also accounts for the volume of CSF included in the tissue compartment by misclassification of CSF-representing voxels as tissue voxels due to the automatic tissue-CSF segmentation (see below) or to the partial volume effect from CSF in Virchow-Robin spaces along penetrating arterioles. The third parameter is analogous to the blood volume fraction in conventional tracer kinetic analysis of brain tissue with vascular space. The observed tracer concentration in the tissue compartment $\left(M^{\prime}{ }_{\mathrm{B}}(t)\right)$ is therefore a combination of the CSF and brain tissue tracer concentrations (Fig. $1 f$ ), that is:

$$
M_{B}^{\prime}(t)=\left(1-V_{\mathrm{CSF}}\right) \cdot M_{\mathrm{B}}(t)+V_{\mathrm{CSF}} \cdot C_{\mathrm{CSF}}(t) .
$$

Isolating $M_{\mathrm{B}}(t)$ in Equation 3 and substituting it and its first derivative with respect to $t$ in Equation 1, the two-compartment model of wholebrain analysis extends to the following:

$$
\begin{aligned}
\frac{d M_{\mathrm{B}}^{\prime}(t)}{d t}=K_{1} \cdot\left(1-V_{\mathrm{CSF}}\right) \cdot C_{\mathrm{CSF}}(t)-k_{2} \cdot\left(M_{\mathrm{B}}^{\prime}(t)\right. & \left.-V_{\mathrm{CSF}} \cdot C_{\mathrm{CSF}}(t)\right) \\
& +V_{\mathrm{CSF}} \cdot \frac{\mathrm{dC}_{\mathrm{cdf}}(t)}{d t},
\end{aligned}
$$

with the three parameters $K_{1}, k_{2}$, and $V_{\mathrm{CSF}}$.

The kinetic model measures the transport of tracer solution, not CSF itself. As in other studies of the glymphatic system using tracers, the model assumes that Gd-DOTA flow reflects CSF-ISF flow and exchange, and that this reflection is invariant in the disease studied here. Equation 4 is a one-compartment first-order rate equation that models transport process from CSF to the brain tissue compartment and does as such not allow discrimination between convection and diffusion.

To avoid confusion between the terminologies of classical kinetic analysis and glymphatic analysis, in the latter of which the term "clearance" is used to denote removal of fluids with dissolved macromolecules from the brain, we refer to $K_{1}$ as the "influx rate" and $k_{2}$ as the "efflux rate constant."

\section{Animals}

Male normotensive Wistar Kyoto rats (WKY; https://www.criver.com/ products-services/find-model/wistar-kyoto-wky-rat) and SHR rats (https://www.criver.com/products-services/find-model/spontaneouslyhypertensive-shr-rat) were obtained from Charles River. The hypertensive strain was developed and first described by Okamoto and Aoki (1963) and remains the most commonly used animal model for cardiovascular disease due to its close resemblance to progressive hypertension in humans (Doggrell and Brown, 1998). Rats are prehypertensive until 6-8 weeks of age, whereupon their systolic blood pressure increases rapidly, finally attaining $200-220 \mathrm{mmHg}$ at $\sim 14$ weeks of age (Adams et al., 1989). The rats were housed in individually ventilated cages with ad libitum access to water and food, and a 12/12 h light cycle from 6:00 A.M. to 6:00 P.M., with $30 \mathrm{~min}$ of twilight at each transition. All treatments and imaging were performed according to protocols approved by the Danish Animal Experiments Inspectorate. Separate groups of rats were scanned at two age ranges: young (7-9 weeks old; $y W K Y, n=11$; and ySHR, $n=$ 8 ) and adult (19-21 weeks; aWKY, $n=8$; and aSHR, $n=9)$. The young and adult SHR groups represent the emergent, early hypertension, and chronic phases of hypertension, respectively.

\section{MRI}

All MRI investigations were conducted on a 9.4 T magnet (Bruker BioSpec 9.4/30 USR) interfaced to a Bruker Advance III console and controlled by Paravision 5.1 software (Bruker BioSpin) at the Faculty of Health and Medical Sciences, University of Copenhagen. Imaging was performed with an $86 \mathrm{~mm}$ (ID) volume resonator and a 4-channel surface quadrature array receiver coil.

\section{Experimental design}

Rat imaging. Surgical anesthesia was induced with 3\% isoflurane in oxygen, followed by a single intraperitoneal injection of a mixture containing ketamine $(100 \mathrm{mg} / \mathrm{kg})$, dexmedetomidine (DEXM; $0.25 \mathrm{mg} / \mathrm{kg}$ ), and glycopyrrulate $(0.2 \mathrm{mg} / \mathrm{kg})$, and supplemented with $1.0 \%-1.5 \%$ isoflurane for maintenance. Using a stereomicroscope (Leica Microsystems, MZ6), the atlanto-occipital membrane was exposed. A drop of $20 \%$ aluminum chloride (Sigma-Aldrich) in water was placed on the incision and 
the exposed skull to stop bleeding and improve the subsequent curing of cyanoacrylate glue following catheter insertion. The intracisternal catheter was prepared by inserting a segment of PE10 tubing $(2.5 \mathrm{~cm}$ long, $0.28 \mathrm{~mm}$ ID $\times 0.61$ $\mathrm{mm}$ OD, Braintree Scientific) inside a pulled glass capillary and gluing it in place $(1 \mathrm{~cm}$ long, $0.68 \mathrm{~mm}$ ID $\times 1 \mathrm{~mm}$ OD aluminosilicate micropipette; Sutter Instruments), with the tip of the glass needle having an ID of $\sim 150 \mu \mathrm{m}$. The catheter was inserted 2-3 mm deep in the CM and glued in place using cyanoacrylate glue. To improve delivery of the tracer to the brain (Lee et al., 2015), the animals were placed in a supine position within the bore of the magnet, and the CM cannula connected to a $100 \mu \mathrm{l}$ Hamilton syringe mounted in a microinfusion pump (Legato 130, KD Scientific) for the delivery of contrast agent $(20 \mu \mathrm{l}, 13.5 \mathrm{~mm}$ GdDOTA at $1.5 \mu \mathrm{l} / \mathrm{min}$ to a total of $0.27 \mu \mathrm{mol}$ Gd-DOTA; Guerbet).

During MR imaging, anesthesia was maintained with the highly selective $\alpha_{2}$-adrenergic receptor agonist DEXM with negligible respiratory and cardiovascular side effects $(0.015-0.020 \mathrm{mg} / \mathrm{kg} / \mathrm{h})$, delivered via a subcutaneous catheter placed in the flank, and $0.6 \%-1 \%$ isoflurane, delivered via a face mask. The use of isoflurane alone for maintaining anesthesia is known to be problematic in studies of SHR and WKY rats because of its cerebrovascular and systemic hemodynamic effects; isoflurane reduces blood pressure in both strains and directly dilates cerebral vasculature (Bencze et al., 2013), while also eliminating the strain difference in blood pressure in adult rats (Leoni et al., 2011). By using DEXM in conjunction with a relatively low concentration of isoflurane, we attempted to minimize potential experimental confounds arising from anesthesia with pure isoflurane. Furthermore, a DEXM/0.8\% isoflurane combination, in contrast to $2 \%$ isoflurane, was recently shown to enhance glymphatic transport function and produce $9-15 \mathrm{~Hz}$ spindle oscillations compared with the high concentration isoflurane, thus mirroring a natural sleep state (Benveniste et al., 2017). Physiological parameters, including respiratory rate, oxygen saturation, heart rate, and rectal body temperature, were continuously monitored using an MRIcompatible monitoring system (SA Instruments). Body temperature was kept within the range of $36.5^{\circ} \mathrm{C}-37.5^{\circ} \mathrm{C}$ using a temperature-controlled waterbed, and physiological parameters were maintained by ongoing adjustments of waterbed temperature, DEXM infusion rate, and isoflurane concentration guided by vital signs.

Imaging parameters. The DCE-MRI protocol was adapted from previous work (Lee et al., 2018) to produce a quantitative and dynamic measurement of gadolinium concentration in the brain after injection of the contrast agent into the CM (for an overview, see Fig. 2). First, we acquired a map of the RF transmission inhomogeneity $\left(\mathrm{B}^{+}\right)$using a double-angle (Stollberger and Wach, 1996) rapid acquisition with relaxation enhancement (RARE) sequence (TE: $22 \mathrm{~ms}$, TR: $10 \mathrm{~s}$, number of averages [NA]: 1, RARE factor: 4, 50 slices, in-plane resolution: $0.24 \times$ $0.24 \mathrm{~mm}$, slice thickness: $0.4 \mathrm{~mm}$; slice gap: $0.2 \mathrm{~mm}$; flip angles [FA]: $70^{\circ}$ and $140^{\circ}$ ), and a precontrast $3 \mathrm{D} \mathrm{T}_{1}$ map was acquired using a variable $\mathrm{FA}$ spoiled gradient method (VFA-SPGR) (TE: $4 \mathrm{~ms}$, TR: $15 \mathrm{~ms}$, NA: 1; matrix: $128 \times 128 \times 128$, voxel size: $0.24 \times 0.24 \times 0.26 \mathrm{~mm}[0.015$ $\mathrm{mm}^{3}$ ], FA: $2,5,10,15,20$, and $30^{\circ}$, scan time per FA: 4:05 min). Then, a series of SPGR images (FA: $15^{\circ}$, otherwise same parameters as VFASPGR) was acquired, consisting of three baseline scans followed by 40 contrast-enhanced scans; each acquisition lasted $4 \mathrm{~min}$ and $5 \mathrm{~s}$ to a total scanning time of $\sim 160 \mathrm{~min}$. A reference phantom filled with $0.1 \mathrm{~mm}$ Gd-DOTA was placed in the FOV to allow intensity normalization between each scan as well as allowing receiver gain adjustment. Reference TX power and receiver gain were kept the same during the scanning session after calculation during the first RARE experiment.

For visualization purposes, we scanned additional 9 young animals ( 3 SHR and $6 \mathrm{WKY}$ ) with high-resolution, long-echo time T2-weighted TURBORARE sequence (TE: 36 ms, TR: 16 s, NA: 3, RARE factor: 8, 128 slices, slice thickness: $0.312 \mathrm{~mm}$; slice overlap: $0.156 \mathrm{~mm}$, matrix: $384 \times$ 384, in-plane resolution: $0.09 \times 0.09 \mathrm{~mm}) 1$ week before DCE-MRI. For this procedure, anesthesia was induced with $3 \%$ isoflurane and maintained with $1.5 \%$ isoflurane. The long echo time produced a high contrast between water and brain tissue, allowing CSF and large PVSs to be delineated from brain.

Blood pressure measurements. To validate that hypertension in the SHR was not affected by the anesthesia, we measured blood pressure in 5 young WKY, 5 young SHR, 4 adult WKY, and 3 adult SHR under the anesthetic regimen of of $3 \%$ isoflurane, $100 \mathrm{mg} / \mathrm{kg}$ ketamine, $0.25 \mathrm{mg} / \mathrm{kg}$ DEMX (induction), and $0.015-0.020 \mathrm{mg} / \mathrm{kg} / \mathrm{h}$ DEXM, $0.6 \%-1 \%$ isoflurane (long-term anesthesia). Blood pressure was measured as the median blood pressure 15-45 min after switch to long-term anesthesia. A 26 weeks of age, SHR was included in the adult SHR group. Blood pressures in all rats were measured through the femoral artery as described by Ghali (2017).

\section{Spatial normalization and segmentation}

The MRI series were corrected for motion by a rigid body registration procedure and resampled into the coordinates for individual mean baseline images. For the purpose of spatial image registration, the image bias field created by the receiver coil was removed using a combination of in-house software and Advanced Normalization Tools (ANTs N4 bias correction) (Tustison et al., 2010), and baseline images were then skullstripped using 3D-PCNN (Chou et al., 2011) followed by a manual correction. Bias field-corrected, skull-stripped baseline images were then used to create a population-based template consisting of the minimum deformation spatial average, as previously described (Avants et al., 2011). The images were rigidly registered to a randomly selected individual image from the series and averaged to create an intermediate template. This process was repeated using the intermediate template resulting from the previous iteration, using first affine and then nonlinear registration steps until the difference between iterations was negligible. Finally, all mean baseline SPGR images were nonlinearly registered to the population-based template and the calculated transformation applied to the SPGR time series, allowing comparisons in a common space, "template space." To allow segmentation of individual time series, the Waxholm Space atlas of the Sprague Dawley rat brain (Papp et al., 2014) was nonlinearly registered to template space and subsequently transformed to the space of the SPGR time series. All registration procedures were performed using the ANT software package version 2.1.0 (RRID: SCR_004757). The skull-stripped images were segmented into CSF and brain compartments using ANTs Atropos, with the bias-field corrected baseline images and calculated $\mathrm{T}_{1}$ maps serving as inputs.

\section{Data preparation}

Signal enhancement in the post-contrast frames due to the infusion of Gd-DOTA was calculated from the motion-corrected series of SPGR images as maps of percent signal increase from baseline (enhancement ratio $[\mathrm{ER}]$ ) and concatenated into a $4 \mathrm{D}$ time series for each animal. 
Subsequently, the ER time series were transformed into units of GdDOTA concentration ([Gd]) as described by Lee et al. (2018). Each voxel in the resulting [Gd] map has a value corresponding to Gd-DOTA "concentration" (i.e., mass of Gd-DOTA per volume), but since said volume does not correspond to the aqueous solution of the tracer, we report the [Gd] maps in units of $\mu \mathrm{mol} / \mathrm{cm}^{3}$ to avoid confusion.

\section{CSF flow patterns and ventricular morphometry}

To characterize gross CSF flow differences between the groups, we calculated an average [Gd] map time series in the template space, by averaging the $[\mathrm{Gd}]$ maps for each group for each MRI time point. This resulted in a mean $[\mathrm{Gd}]$ time series for each of the four groups (yWKY, ySHR, aWKY, and aSHR). As reflux of CSF through the cerebral aqueduct back into the ventricular system is a well-described phenomenon in normal pressure hydrocephalus (Kim et al., 1999; Vanneste, 2000; Qvarlander et al., 2017), we quantified ventricular reflux by calculating the total mass of Gd-DOTA contained in the lateral ventricles (LVs) $40 \mathrm{~min}$ after CM injection of Gd-DOTA. The LVs and CM were manually delineated based on the previously calculated CSF segmentation using ITKSNAP software version 3.6, and total ventricular Gd mass (in nmol) was then calculated as the sum of [Gd] in all ventricular voxels multiplied by the volume per voxel, $0.015 \mathrm{~mm}^{3}$.

Quantification of whole-brain glymphatic influx and efflux Hydrocephalus with CSF space enlargement as well as reduced brain volume are well-characterized innate features of SHR rats (Tajima et al., 1993; Martínez-Peña y Valenzuela et al., 2006). The anatomical CSF features of SHR rats change the overall CSF flow patterns and must be accommodated when applying the methods normally used to measure glymphatic influx and clearance in DCE-MRI (Lee et al., 2015; Jiang et al., 2017; Ringstad et al., 2017).

To evaluate global glymphatic function in the SHR, we applied kinetic modeling to CSF and brain TCCs as described above. From the individual [Gd] time series, we computed TCCs as the average Gd-DOTA concentration over time in CSF and brain, respectively, in the native space images of individual rats using the segmentations of CSF and brain previously calculated. Using a gradient-expansion algorithm as implemented in iFit software version 0.82 (http://www.liver.dk/ifit.html), a one-tissue compartment model was fitted to the data for estimating the influx rate from CSF to brain $\left(K_{1} ; \mathrm{ml} \cdot \mathrm{cm}^{-3} \cdot \mathrm{min}^{-1}\right)$ and the efflux rate constant from brain $\left(k_{2} ; \mathrm{min}^{-1}\right)$. To account for CSF included in the brain compartment, the CSF volume fraction $\left(V_{\mathrm{CSF}} ; \%\right)$ was included in the model fit (Eqs. 3, 4).

\section{Quantification of local glymphatic influx}

Hydrocephalus and CSF reflux inevitably impact total tracer mass distribution before it enters the glymphatic pathway. Since the impact of CSF space enlargement on the availability of tracers to the glymphatic system in the whole-brain kinetic analysis is unknown, we further validated the results derived from the global glymphatic analysis in compartments not affected by the innate hydrocephalus of SHR. We modeled the local transport of Gd-DOTA from the PVS of the MCA into the cerebral cortex (CTX) immediately medial to the MCA. Specifically, we measured TCCs in two ROIs drawn manually in the native space images of individual rats: in each transverse image slice between the circle of Willis $(\mathrm{CoW})$ and the rostral rhinal vein, the PVS was defined as a $3 \times 3$ voxel square centered in the MCA but excluding nonbrain voxels. The CTX was defined as a disc of six voxels diameter located directly medial-posterior to PVS. Due to high noise levels in the [Gd] images maps along the brain-skull interface, we instead used ER maps for this calculation.

We then fitted a one-tissue compartment model to the PVS and CTX TCCs and calculated the magnitude of $K_{1}\left(\mathrm{ml} \cdot \mathrm{cm}^{-3} \cdot \mathrm{min}^{-1}\right)$ and $k_{2}$ $\left(\mathrm{min}^{-1}\right)$ using iFit software version $0.82 . K_{1}$ for each animal was measured as the average of $K_{1}$ measured in the right and left hemispheres, as for $k_{2}$. Care was taken when delineating the CTX compartment not to include CSF in the drawn ROIs wherefore the CSF-volume parameter as the MCA ROIs was not included in this analysis (Eq. 1). Since the CTX compartment is contiguous with the surrounding brain tissue, the tracer passes through the compartment without a well-defined destination. Thus, the efflux rate constant from the CTX compartment, $k_{2}$, is not a meaningful measure of glymphatic efflux in the context of the local glymphatic analysis.

\section{Gd-DOTA transport in white matter}

We additionally evaluated tracer kinetics in the corpus callosum in three young SHR and 6 young WKY rats. We could only delineate the corpus callosum in those animals for which we had acquired additional $\mathrm{T}_{2}-$ weighted TURBORARE images, which provided excellent anatomical contrast. Corpus callosum was delineated manually using ITK-SNAP software version 3.6, and TCCs were calculated from the dynamic [Gd] images.

\section{Immunohistochemistry}

The effects of astrogliosis and AQP4 expression in the astrocytes were also evaluated in the present study to assess their putative correlation to the glymphatic CSF-to-brain clearance. A subset of the young (yWKY, $n=3$; ySHR, $n=3$ ) and adult rats (aWKY, $n=3$; aSHR, $n=3$ ) were after the DCE-MRI experiment anesthetized by intraperitoneal injection of a mixture of ketamine and xylazine $\left(100 \mathrm{mg} \mathrm{kg}^{-1} / 10 \mathrm{mg} \mathrm{kg}^{-1}\right)$ and perfused transcardially with $100 \mathrm{ml} 0.01 \mathrm{M}$ PBS, pH 7.4 (Sigma-Aldrich) containing heparin (10,000 units/L; SAI Infusion Technologies). Vasculature was labeled by perfusing $10 \mathrm{ml}$ of a solution containing $100 \mu \mathrm{g} / \mathrm{ml}$ wheat lectin (Triticum vulgaris) conjugated to FITC (Sigma-Aldrich), and followed by $140 \mathrm{ml}$ 4\% PFA solution (Sigma-Aldrich), both diluted in $0.01 \mathrm{~m} \mathrm{PBS}$, pH 7.4. Brains were dissected, postfixed overnight in $4 \%$ PFA at $4^{\circ} \mathrm{C}$, and cryopreserved by incubation in sucrose solutions of $10 \%$, $20 \%$, and $30 \%$, diluted in $0.01 \mathrm{~m} \mathrm{PBS}, \mathrm{pH} 7.4$. Rat brains were embedded in optimal cutting temperature compound (Sigma-Aldrich) and frozen in isopentane on dry ice; $30 \mu \mathrm{m}$ sections were obtained using a cryostat (Leica Microsystems, CM186). After PBS washes, histological sections were blocked for $1 \mathrm{~h}$ at room temperature in a solution containing $0.3 \%$ Triton X-100 (Sigma-Aldrich) and 5\% normal donkey or goat serum (Invitrogen; Thermo Fisher Scientific) diluted in 0.01 м PBS, pH 7.4, followed by overnight incubation at $4^{\circ} \mathrm{C}$ with the following primary antibodies diluted in blocking solution: polyclonal chicken anti-GFAP (1: 500, Thermo Fisher Scientific) and polyclonal rabbit anti-AQP4, C terminus (1:500, Merck). Immunolabeling was revealed by incubation with the appropriate secondary antibodies coupled to fluorophores (AlexaFluor, 1:500; Invitrogen; Thermo Fisher Scientific) for $2 \mathrm{~h}$ at room temperature. DAPI ( $1 \mu \mathrm{g} / \mathrm{ml}$; Thermo Fisher Scientific) was used for nuclear counterstaining, and slides were mounted with Prolong Gold Antifade Reagent (Invitrogen; Thermo Fisher Scientific). Images of the immunolabeled slides were acquired on a confocal microscope (Nikon Eclipse Ti) with Plan Fluor $20 \times / 0.75 \mathrm{Mlmm}$ or an epifluorescence microscope (Nikon Ni-E) with Plan Apo $\lambda 4 \times / 0.20$ objective. Confocal imaging acquisition was manually corrected to equalize the lectin signal across the optical sections. For the wavelengths corresponding to the signal of GFAP and AQP4, laser power and digital gain were kept the same for all acquired images, for both WKY and SHR samples. Acquired images were adjusted for brightness and contrast using FIJI/ImageJ software.

\section{Statistical analysis}

Measurements are reported as mean \pm SD. Unless noted, statistical tests were performed as one-tailed (in cases with a hypothesized direction of change, e.g., decreased influx rate in SHR) or two-tailed Mann-Whitney $U$ tests. Analyses were conducted using Prism 7, MATLAB 9.3 (RRID: SCR_001622), and image scripting was performed in Python version 2.7.10 (RRID:SCR_008394). 3D volume rendering was created using MRIcro version 1.8.9 (http://www.mccauslandcenter.sc.edu/crnl/mricro) software.

\section{Results}

Image series from 6 rats were excluded from the analysis due either to failed infusion of Gd-DOTA (3 rats) or to premature termination of the experiment for technical reasons (3 rats). Additionally, quantification of the Gd-DOTA concentration ([Gd]) failed in three experiments due to suboptimal coil placement; these experiments were excluded from analyses requiring the 
Table 1. Summary of weights and average heart and respiration rates during scan (mean \pm SD)

\begin{tabular}{llllll}
\hline $\begin{array}{l}\text { Age } \\
\text { (wk) }\end{array}$ & Strain & $N($ excluded) & Weight $(\mathrm{g})$ & $\begin{array}{l}\text { Heart } \\
\text { rate }(\mathrm{min})\end{array}$ & $\begin{array}{l}\text { Respiratory } \\
\text { rate (min) }\end{array}$ \\
\hline $7-9$ & WKY & $9(2)$ & $245 \pm 16$ & $251 \pm 23$ & $61 \pm 6$ \\
& SHR & $7(1)$ & $216 \pm 23$ & $275 \pm 30$ & $59 \pm 5$ \\
$19-21$ & WKY & $6(2)$ & $366 \pm 9$ & $238 \pm 26$ & $68 \pm 5$ \\
& SHR & $8(1)$ & $338 \pm 31$ & $252 \pm 12$ & $56 \pm 7^{* * a}$ \\
\hline
\end{tabular}

${ }^{* *} p<0.01$ (two-tailed $t$ test with unequal variance: $y W K Y$ vs ySHR and aWKY vs aSHR, followed by Bonferroni correction for multiple comparisons); ${ }^{a} p=0.0067, \mathrm{df}=11, t=3.81$.

Table 2. Summary of results from the blood pressure measurements under the MRI anesthesia regimen

\begin{tabular}{lllc}
\hline Age (wk) & Strain & $N$ & BP $(\mathrm{mmHg})$ \\
\hline $7-9$ & WKY & 5 & $78.8 \pm 14.5$ \\
& SHR & 5 & $119.4 \pm 10.6^{* * a}$ \\
$19-21$ & WKY & 4 & $72.1 \pm 5.5$ \\
& SHR & 3 & $139.3 \pm 13.6^{* \mathrm{~b}}$ \\
\hline
\end{tabular}

${ }^{*} p<0.05 ;{ }^{* *} p<0.01$; one-tailed Mann-Whitney $U$ tests (SHR $>$ WKY); ${ }^{a} p=0.004 ;{ }^{b} p=0.029$.

[Gd] time series but were included in other analyses. All rats included in analyses were physiologically stable for the duration of the scan. Body weights as well as average heart rate and respiratory rate during the scanning period are summarized in Table 1 , along with statistical comparisons within age groups ( $y W K Y$ vs ySHR, and aWKY vs aSHR). Mean respiratory rates were significantly lower in the aSHR group compared with the normotensive age-matched controls.

\section{Blood pressure under anesthetic regimen}

Blood pressures measured in 5 young WKY, 5 young SHR, 4 adult WKY, and 3 adult SHR under the anesthetic regimen for MRI are reported in Table 2. Blood pressure was significantly higher in SHR than WKY in both age groups despite anesthesia.

\section{Morphological brain changes in SHR rats}

Hydrocephalus and reduced brain volume were evident in our cohorts of both young and adult SHRs compared with WKYs (Fig. $3 b-d$; summarized in Table 3 ). The intracranial volumes and brains of the SHR rats were significantly smaller than their normotensive controls (Fig. $3 b$ ), with 11\% lower brain volume in the young group and $12 \%$ lower in the adult group. As expected, ventricular volumes were higher in SHRs compared with WKYs (Fig. $3 c, d$ ), with fivefold higher LV volumes in the young SHRs and sixfold higher LV volumes in the adults, as well as a $21 \%$ higher CM volume in the young group and 49\% higher CM volume in the adult group (Table 3). Surprisingly, total CSF volume (including subarachnoid space CSF and ventricular system) did not differ significantly between the young SHR and WKY, and nonventricular CSF volume (excluding lateral, third, and fourth ventricles) was indeed 7\% lower in the young SHR (Table 3). Conversely, total CSF volume was $8 \%$ greater in adult SHR than their age-matched controls, although no difference was found in nonventricular CSF volume (Table 3). Calculated as a percentage of intracranial volume, total CSF volume was higher in SHR in both age groups, whereas the relative nonventricular CSF volume was greater in the adult group of SHR but did not differ in the young group (Table 3). Small CSF spaces (e.g., the subarachnoid space) are smaller than the resolution of the SPGR, and thus CSF volumes are likely to be overestimated. Since the segmentation was automatic, this overestimation was not likely to be biased in either strain.
To further elucidate the differences in nonventricular CSF volumes, we measured the volumes of the CSF-filled subarachnoid space surrounding CoW and the basal part of the brainstem, as these are major passageways for tracer from CM to the brain. As we worried that the spatial resolution of the SPGR images was too low to precisely measure these volumes, we manually delineated them using the higher-resolution, higher CSF-contrast TURBORARE images, which were only acquired for 3 young SHR and 6 young WKY. We found no significant difference in subarachnoid CSF volume surrounding CoW (yWKY, $6.17 \pm$ $0.25 \mathrm{~mm}^{3}$; ySHR, $5.71 \pm 0.31 \mathrm{~mm}^{3} ; p=0.095$, two-tailed MannWhitney $U$ test), but subarachnoid CSF volume was significantly lower in basal brain stem (yWKY, $0.76 \pm 0.03 \mathrm{~mm}^{3}$; ySHR, $0.57 \pm 0.07 \mathrm{~mm}^{3} ; p=0.024$, two-tailed Mann-Whitney $U$ test).

\section{Changes in CSF flow patterns in SHR rats}

We qualitatively evaluated the transport of Gd-DOTA from CM into the cerebral ventricles by volume rendering the group mean [Gd] time series (Fig. 3e,f). In the ySHR group, Gd-DOTA reached the fourth ventricle $(4 \mathrm{~V})$, third ventricle $(3 \mathrm{~V})$, and LVs at 12,24 , and $32 \mathrm{~min}$, respectively, after injection. In the average yWKY group, the contrast agent did not reflux into the ventricles but was instead transported dorsally from the CM over the cerebellar vermis to the pineal recess, making its first appearance at 8 min after injection in the average yWKY group but not in the average ySHR group. Similar flow characteristics were observed in the adult groups: contrast agent was apparent in the $4 \mathrm{~V}$ at 8 min after injection, in $3 \mathrm{~V}$ at $16 \mathrm{~min}$ and in LV $24 \mathrm{~min}$ after injection in the average aSHR, with no contrast agent being observed in the ventricular system of the aWKY group.

To quantify ventricular reflux, we measured the total mass of contrast agent that accumulated in the LV at 40 min after injection (Fig. $3 g$ ). We observed 10-fold more Gd-DOTA in the LV of ySHR compared with controls ( $y W K Y, 0.11 \pm 0.07 \mathrm{nmol}$; ySHR, $1.42 \pm 1.62 \mathrm{nmol} ; p=0.006)$, and 30-fold more Gd-DOTA in the adult SHR rats $($ aWKY, $0.10 \pm 0.13 \mathrm{nmol}$; aSHR, $3.48 \pm 3.96$ $\mathrm{nmol} ; p=0.0025)$. Regardless of age, transfer of contrast agent to LV was considerably more variable in SHR than in WKY rats.

\section{Effects of hypertension on the global glymphatic transport}

To calculate kinetic glymphatic parameters, we first computed TCCs from CSF and brain, respectively (Fig. 4), using the dynamic parametric [Gd] maps, available in $9 \mathrm{yWKY}, 6 \mathrm{ySHR}, 5$ aWKY, and 7 aSHR; average TCCs for both CSF and brain are shown in Figure $4 a-d$.

Peak average $[\mathrm{Gd}]$ in brain tissue did not differ significantly in the young group (yWKY, $0.042 \pm 0.005 \mathrm{mmol} / \mathrm{cm}^{3}$; $\mathrm{ySHR}$, $\left.0.037 \pm 0.003 \mathrm{mmol} / \mathrm{cm}^{3} ; p=0.11\right)$, but was $20 \%$ lower in the adult SHR compared with WKY (aWKY, $0.039 \pm 0.007 \mathrm{mmol} /$ $\mathrm{cm}^{3}$; aSHR, $0.031 \pm 0.004 \mathrm{mmol} / \mathrm{cm}^{3} ; p=0.018$; two-tailed Mann-Whitney $U$ test). Peak total mass of Gd in brain tissue was $22 \%$ lower in young SHR ( $\mathrm{yWKY}, 79.4 \pm 9.7 \mathrm{nmol}, 29.4 \pm 3.6 \%$ of the injected dose $\left[\%_{\mathrm{ID}}\right]$; ySHR, $61.8 \pm 4.9 \mathrm{nmol}, 22.9 \pm$ $1.8 \%_{\mathrm{ID}} ; p=0.0004$ ) and $28 \%$ lower in adult SHR (aWKY, $80.3 \pm 14.1 \mathrm{nmol}, 29.7 \pm 5.2 \%_{\mathrm{ID}} ; \mathrm{aSHR}, 57.6 \pm 5.2 \mathrm{nmol}, 21.3 \pm$ $1.9 \%_{\text {ID }} ; p=0.01$; two-tailed Mann-Whitney $U$ test). No significant differences were found in the time-to-peak (TTP) Gd concentration $(y W K Y, 61.9 \pm 8.4 \mathrm{~min} ; \mathrm{ySHR}, 81.0 \pm 31.7 \mathrm{~min} ; p=$ 0.28; aWKY, $59.2 \pm 10.0 \mathrm{~min}$; aSHR, $65.0 \pm 20.4 \mathrm{~min} ; p=0.90$; two-tailed Mann-Whitney $U$ test).

We then quantified global glymphatic influx and efflux rates using a one-tissue compartment model, including a $V_{\mathrm{CSF}}$ parameter (Fig. $4 e, f$ ). For full results, including root mean square error 
a Example MRI segmentations $\mathbf{b}$

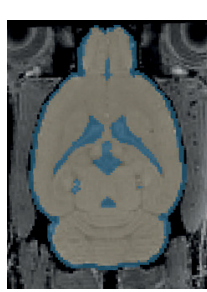

young SHR
ICSF Brain

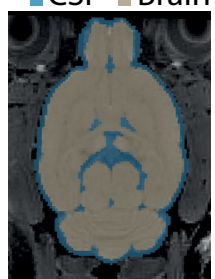

young WKY

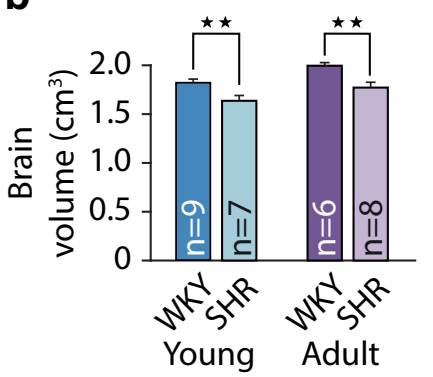

C

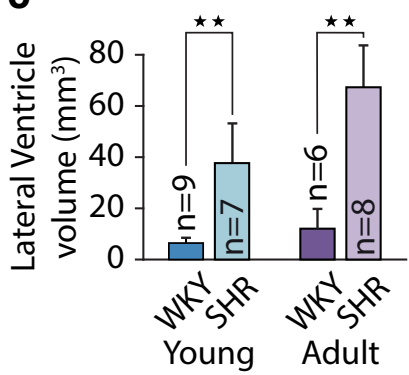

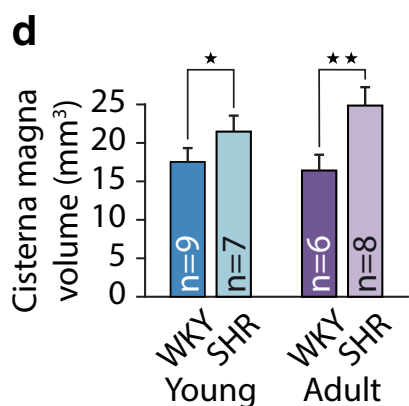

e
Inj. +8 min

Inj. +12 min

Inj. +16 min

Inj. $+24 \min$

Inj. $+32 \mathrm{~min}$
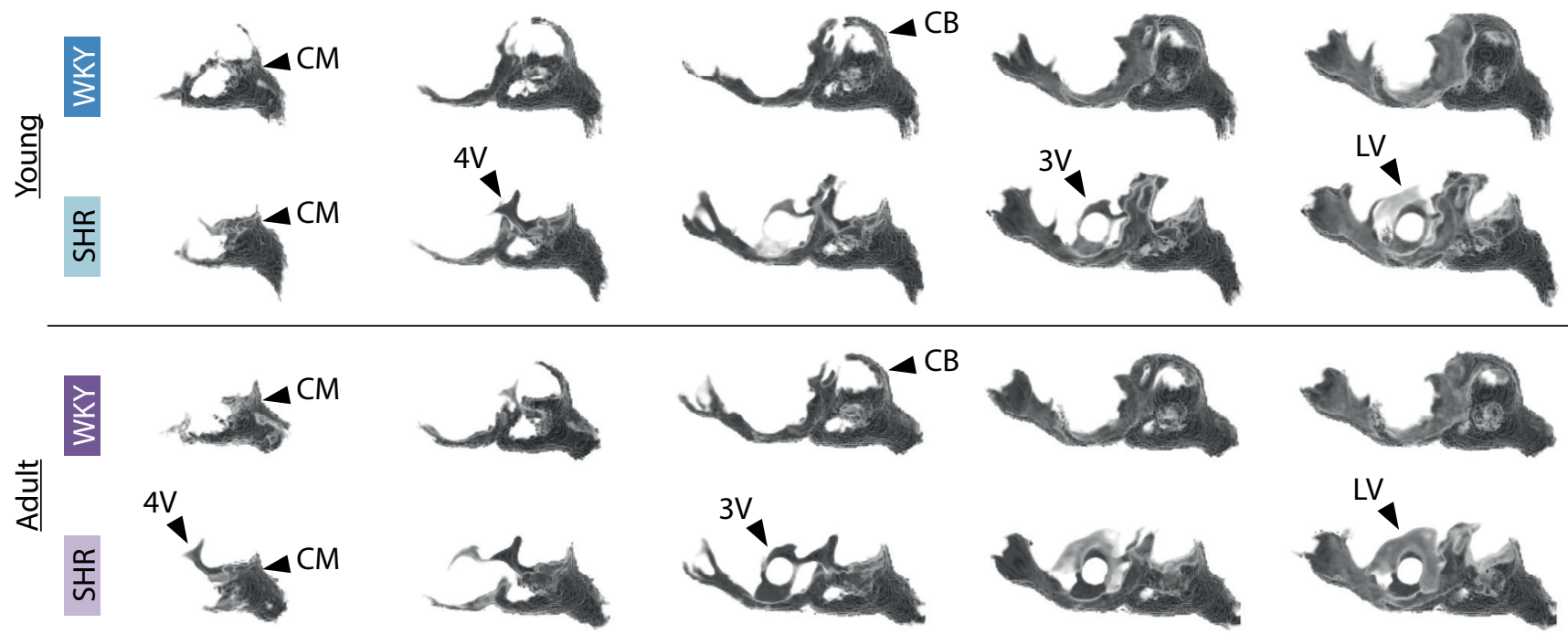

Inj. +16 min

Inj. +12 min

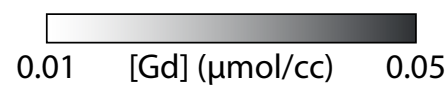

f

Anatomical overview
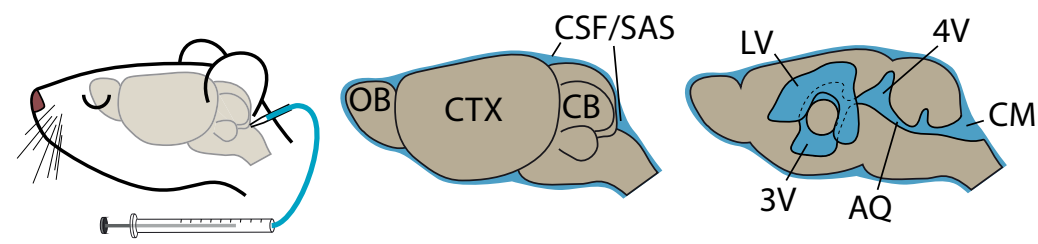

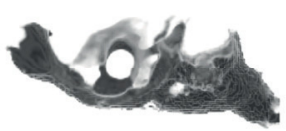

Inj. $+24 \min$

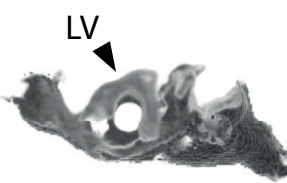

Inj. +32 min

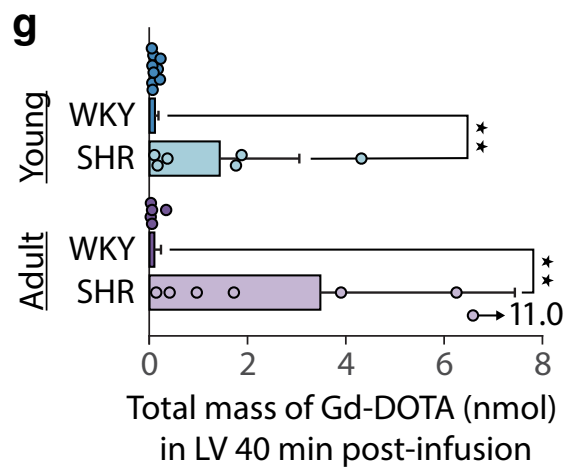

Figure 3. $a$, Gross morphological and CSF flow changes in the SHR compared with WKY. Example segmentations of baseline SPGR images into brain tissue and CSF. Brain volumes were significantly lower in both young and adult SHR than their normotensive controls (b), and the volumes of LVs (c) and CM (d) were significantly higher for the SHR compared with WKY. Gd-DOTA was infused into the CM starting at injection +0 min and continuing for $13 \mathrm{~min} 20 \mathrm{~s}$. $\boldsymbol{e}$, Volume rendering of average Gd-DOTA concentration time series for each of the four groups revealed differences in gross CSF flow. $\boldsymbol{f}$, In both young and adult SHR, Gd-DOTA appeared in the 4V, 3V, and LVs within 32 min after the start of the infusion. Overview of brain and ventricular anatomy. Total mass of Gd-DOTA in the LVs 40 min after the start of infusion was markedly higher in the SHR in both age groups $(\boldsymbol{g}) .{ }^{*} p<0.05,{ }^{* *} p<0.01$. CB, Cerebellum; 0B, olfactory bulb; SAS, subarachnoid space; $A Q$, aqueduct.

Table 3. Summary of brain and ventricle sizes in SHR and WKY

\begin{tabular}{|c|c|c|c|c|c|c|c|c|c|c|}
\hline Age (wk) & Strain & $N$ & Intracranial $\left(\mathrm{cm}^{3}\right)$ & Brain $\left(\mathrm{cm}^{3}\right)$ & $\operatorname{CSF}\left(\mathrm{cm}^{3}\right)$ & $\begin{array}{l}\text { Nonventricular } \\
\operatorname{CSF}\left(\mathrm{cm}^{3}\right)\end{array}$ & $\operatorname{LV}\left(\mathrm{mm}^{3}\right)$ & $\mathrm{CM}\left(\mathrm{mm}^{3}\right)$ & $\operatorname{CSF}\left(\%^{n}\right)$ & $\begin{array}{l}\text { Nonventricular } \\
\text { CSF }\left(\%{ }^{n}\right)\end{array}$ \\
\hline \multirow[t]{2}{*}{$7-9$} & WKY & 9 & $2.45 \pm 0.06$ & $1.89 \pm 0.04$ & $0.56 \pm 0.03$ & $0.55 \pm 0.03$ & $6.5 \pm 1.7$ & $17.7 \pm 1.7$ & $22.7 \pm 0.8$ & $22.3 \pm 0.8$ \\
\hline & SHR & 7 & $2.24 \pm 0.06^{* * * a}$ & $1.69 \pm 0.06^{* * * c}$ & $0.55 \pm 0.04$ & $0.51 \pm 0.02^{* f}$ & $38.6 \pm 16.5^{* * * g}$ & $21.4 \pm 2.2^{* * i}$ & $24.6 \pm 1.5^{* \mathrm{k}}$ & $22.7 \pm 0.8$ \\
\hline \multirow[t]{2}{*}{$19-21$} & WKY & 6 & $2.66 \pm 0.03$ & $2.07 \pm 0.03$ & $0.59 \pm 0.02$ & $0.58 \pm 0.02$ & $9.6 \pm 7.4$ & $16.9 \pm 2.2$ & $22.1 \pm 0.7$ & $21.7 \pm 0.6$ \\
\hline & SHR & 8 & $2.47 \pm 0.07^{* * * b}$ & $1.83 \pm 0.06^{* * * d}$ & $0.64 \pm 0.03^{* * \mathrm{e}}$ & $0.57 \pm 0.01$ & $68.0 \pm 17.4^{* * * h}$ & $25.2 \pm 2.4^{* * * j}$ & $25.9 \pm 1.0^{* * * 1}$ & $22.9 \pm 0.6^{* * m}$ \\
\hline
\end{tabular}

${ }^{*} p<0.05 ;{ }^{* *} p<0.01 ;{ }^{* * *} p<0.001 ;$ two-tailed Mann-Whitney U tests; ${ }^{\mathrm{a}} p=0.00017 ;{ }^{\mathrm{b}} p=0.00067 ;{ }^{\mathrm{c}} p=0.00017 ;{ }^{\mathrm{d}} p=0.00067 ;{ }^{\mathrm{e}} p=0.008 ;{ }^{\mathrm{f}} p=0.012 ;{ }^{9} p=0.00017 ;{ }^{\mathrm{h}} p=0.00067 ;{ }^{\mathrm{i}} p=0.002 ;{ }^{\mathrm{j}} p=0.00067 ;{ }^{\mathrm{k}} p=0.012 ;{ }^{1} p=$ $0.00067 ;{ }^{\mathrm{m}} p=0.0027 ;{ }^{\mathrm{n}}$ percentage of intracranial volume. 

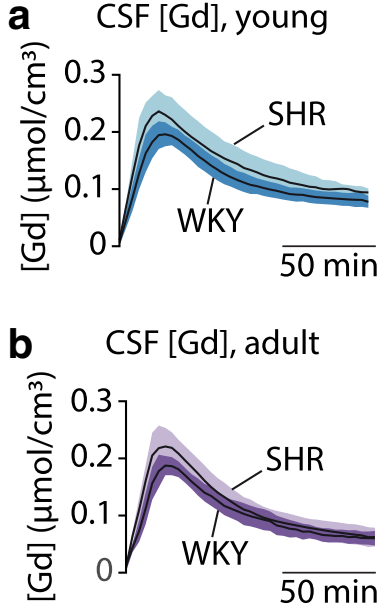

e $\begin{gathered}\text { 1-tissue } \\ \text { compartment model }\end{gathered}$

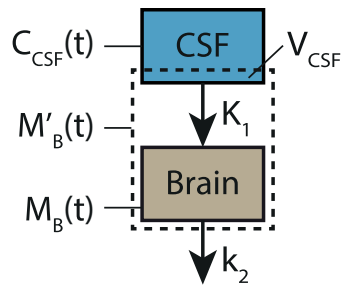

Whole-brain
influx rate
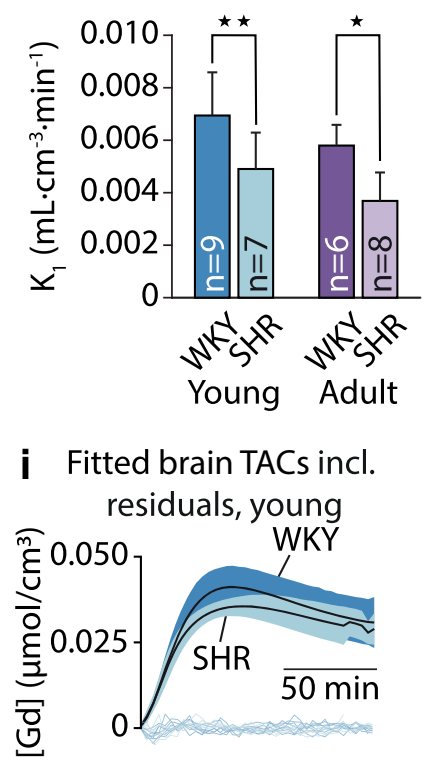
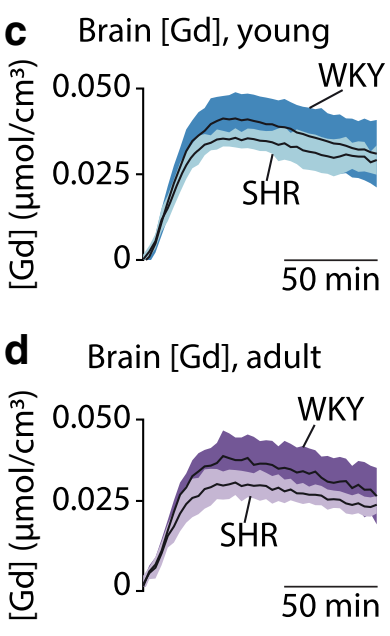

avg. \pm std.

\section{f Example 1-tissue compartment model fit}

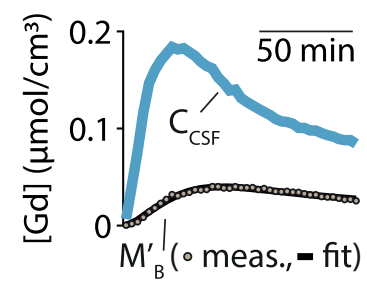

\section{h Whole-brain efflux rate constant}

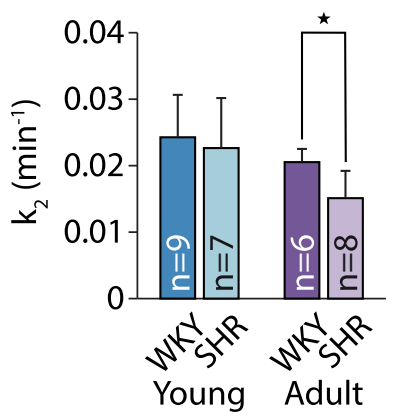

Fitted brain TACs incl. residuals, adult

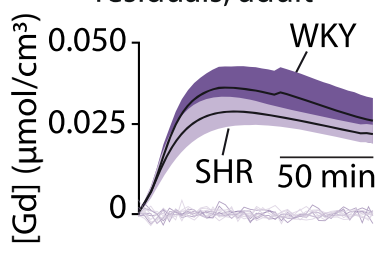

avg. \pm std.

of the fitted model, see Table 4 . The global glymphatic influx rate $\left(K_{1}\right)$ was significantly lower in both young and adult SHR than in corresponding WKY groups (Fig. $4 g$ ), with $29 \%$ lower $K_{1}$ in young SHR and $36 \%$ lower $K_{1}$ in adult SHR. The global glymphatic efflux rate $\left(k_{2}\right)$ was also reduced in adult SHR by $26 \%$, but not significantly in young SHR (Fig. $4 h$ ). The analysis additionally showed a lower volume of distribution $\left(V_{\mathrm{ECS}}\right)$, which is the apparent extracellular space volume fraction in the brain, in the young group by $25 \%$, but not in the adult group. The estimates of CSF volume in the brain tissue compartment calculated from the one-tissue compartment model did not differ significantly between the SHR and WKY groups in either age group, although we observed a tendency toward lower $V_{\mathrm{CSF}}$ in the adult groups. Average modeled TCCs are shown in Figure $4 i, j$ with individual residual errors.

Since CoW is a main passageway for tracer from CM to the brain, we speculated that the decreased glymphatic influx rate was caused by a decreased availability of tracer in the PVS surrounding CoW. To test this, we measured the TTP and area under the curve (AUC) for TACs from subarachnoid CSF surrounding CoW. No differences were found in either TTP (yWKY, $30.6 \pm 8.7 \mathrm{~min}$; $y S H R, 30.6 \pm 5.2 \mathrm{~min} ; p=0.81$; aWKY, $28.9 \pm 4.7 \mathrm{~min}$; aSHR, $31.8 \pm 8.4 \mathrm{~min} ; p=0.72)$ or AUC $(\mathrm{yWKY}$, $48.3 \pm 6.7$; ySHR, 45.2 $\pm 8.8 ; p=0.6 ; \mathrm{aWKY}, 49.2 \pm 11.8$; aSHR, $42.7 \pm 3.3 ; p=0.34$; two-tailed Mann-Whitney $U$ test).

\section{Effects of hypertension on perivascular CSF flow and local glymphatic transport}

To validate the findings of a lowered glymphatic influx rate in whole brain of SHRs independently of changes in gross CSF flow and morphology, we calculated the local tracer transport from the PVS of the MCA to the CTX by fitting a one-tissue compartment model to TCCs from the subarachnoid PVS (input compartment) surrounding the MCA, and adjacent CTX (tissue compartment), thereby deriving the local glymphatic influx rate $K_{1}$ (Fig. 5). ER time series were available for $9 \mathrm{yWKY}, 7 \mathrm{ySHR}, 6$ aWKY, and 8 aSHR animals. $K_{1}$ for each animal was measured as the average of $K_{1}$ measured in the right and left hemispheres, same as for $k_{2}$. Figure $5 a-d$ shows a representative volume rendering of the perivascular flow measured in a yWKY rat overlaid a high-resolution T2-weighted TURBORARE MRI to visualize the PVS. Here, contrast agent traveled along the ventral surface of the brain and reached the PVS surrounding the CoW, from where it followed a perivascular route along the MCA (Fig. 5b). The ROIs for the two compartments are illustrated in Figure $5 d, e$ (which shows an example of the one-tissue compartment model fit to the measured TCCs).

This analysis (for full results, including root mean square error of the fitted model, see Table 5) showed that the mean magnitude of the local glymphatic influx rate $\left(K_{1}\right)$ was significantly lower in adult hypertensive animals by $30 \%$ (Fig. $5 g$ ). No significant difference was identified in the young group, although there was a tendency toward lower average $K_{1}(p=0.105)$. No significant differences of $k_{2}$ were found between the groups (Fig. $5 h$ ). Average modeled TCCs are shown in Figure $5 i, j$ with individual residual errors.

Figure 4. Average TCCs of Gd-DOTA showed occasionally slightly higher CSF Gd-DOTA concentration in the CSF of young $(\boldsymbol{a})$ and adult $(\boldsymbol{b})$ hypertensive rats and their normotensive controls. Average TCCs of Gd-DOTA in the brain compartment of young SHR and WKY (c) and adult SHR and WKY (d). Average TCCs are shown as mean \pm SD. e, Schematic overview of the one-tissue compartment model applied to brain and CSF TCCs to calculate the glymphatic influx rate $\left(K_{1}\right)$ and efflux rate constant $\left(k_{2}\right) . \boldsymbol{f}$, Example of the one-tissue compartment model fit. $\leftarrow$

The calculated $K_{1}$ was significantly lower in the SHR than in WKY in both age groups $(\boldsymbol{g})$, and $k_{2}$ was lower in the adult, but not the young, SHR compared with their age-matched controls $(\boldsymbol{h})$. Average modeled time-activity curves and residuals from the young (i) and adult (j) age groups. ${ }^{*} p<0.05,{ }^{* *} p<0.01$. 
Table 4. Summary of results from the global glymphatic transport analysis

\begin{tabular}{llllllll}
\hline Age $(w k)$ & Strain & $N$ & $K_{1}\left(\mathrm{ml} \cdot \mathrm{cm}^{-3} \cdot \mathrm{min}^{-1}\right)$ & $k_{2}(\mathrm{~min})$ & $V_{\text {ECS }}(\%)$ & $V_{\text {CSF }}(\%)$ & Fit RMSE $\left(\mathrm{mmol} / \mathrm{cm}^{3}\right)$ \\
\hline $7-9$ & WKY & 9 & $0.0069 \pm 0.0017$ & $0.0242 \pm 0.006$ & $29.5 \pm 6.8$ & $5.4 \pm 2.1$ & $0.0016 \pm 0.0011$ \\
& SHR & 6 & $0.0049 \pm 0.0014^{* * a}$ & $0.0226 \pm 0.007$ & $22.0 \pm 2.7^{* \mathrm{~d}}$ & $4.0 \pm 3.1$ & $0.0012 \pm 0.0005$ \\
$19-21$ & WKY & 5 & $0.0058 \pm 0.0008$ & $0.0205 \pm 0.002$ & $28.5 \pm 5.2$ & $6.9 \pm 2.2$ & $0.0012 \pm 0.0001$ \\
& SHR & 7 & $0.0037 \pm 0.0011^{* \mathrm{~b}}$ & $0.0151 \pm 0.004^{* \mathrm{c}}$ & $24.8 \pm 4.9$ & $4.4 \pm 1.7$ & $0.0011 \pm 0.0001$ \\
\hline
\end{tabular}

${ }^{*} p<0.05 ;{ }^{* *} p<0.01 ; K_{1}, k_{2} ;$ one-tailed Mann-Whitney U tests (SHR $<$ WKY); $V_{\mathrm{ESS}}, V_{\text {CSF }}$ : two-tailed Mann-Whitney $U$ tests; ${ }^{a} p=0.0088 ;{ }^{b} p=0.015 ;{ }^{c} p=0.036 ;{ }^{d} p=0.0027$.

a Example of subarachnoid distribution of Gd-DOTA after CM-infusion

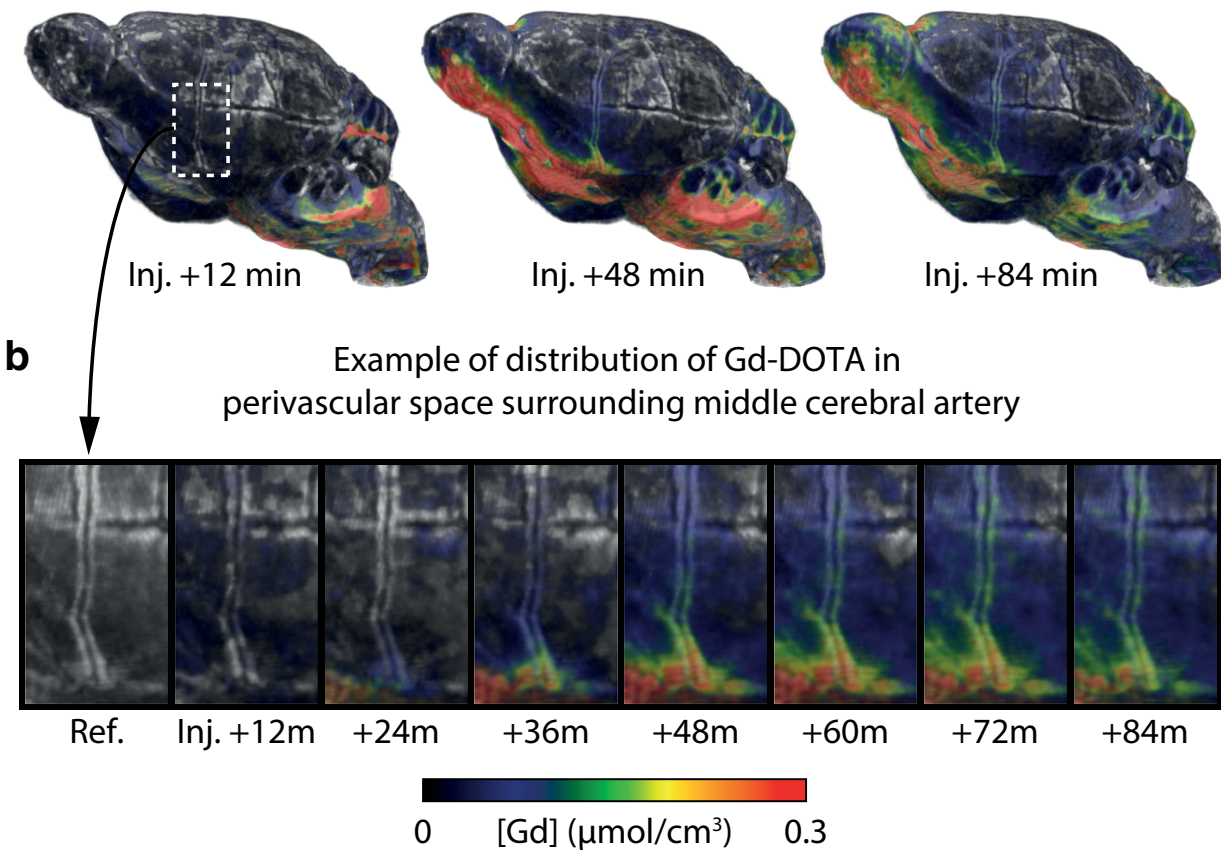

d Location of CTX and PVS ROIs on coronal slice

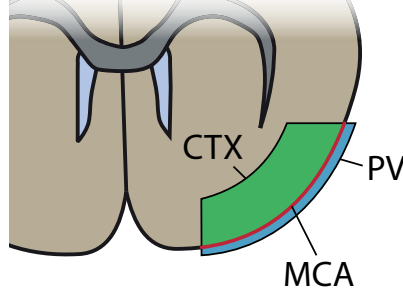

e Local 1-tissue $\mathbf{f}$ compartment model
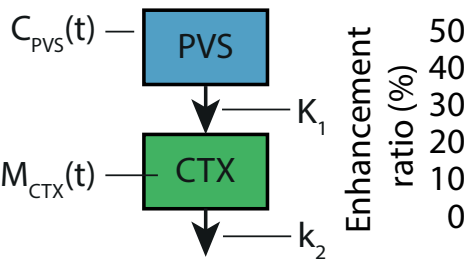

Example 1-tissue compartment model fit

i $\quad$ Fitted CTXTACs incl. residuals, young

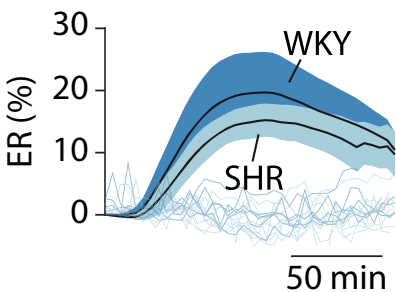

$50 \mathrm{~min}$
C Anatomical reference
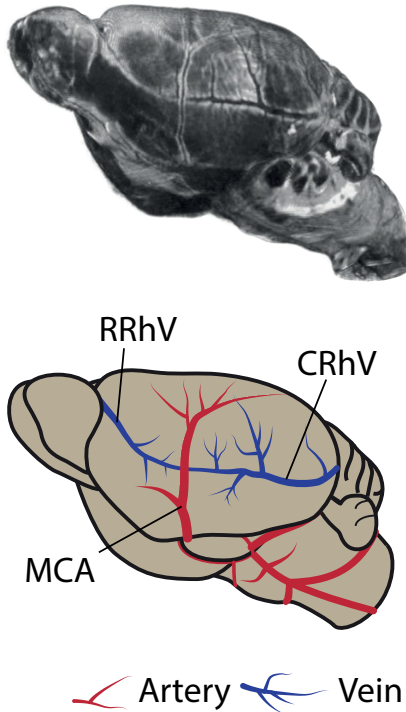

g Local influx rate

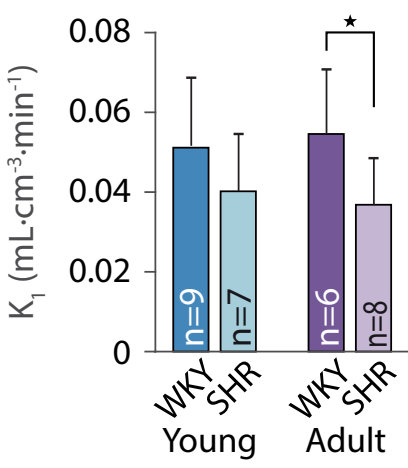

h Local efflux rate constant

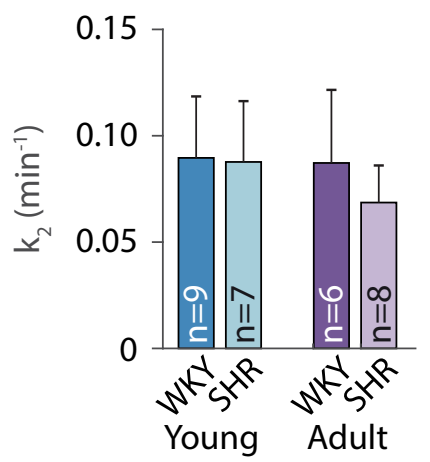

Figure 5. $\boldsymbol{a}$, A representative volume rendering of the CSF tracer Gd-DOTA through the perivascular/subarachnoid spaces on the brain surface of a 7-week-old normotensive Wistar Kyoto rat. $\boldsymbol{b}$, Zoomed view of the CSF tracer traveling up along the PVS surrounding the MCA. $\boldsymbol{c}$, Anatomical and vascular reference for $\boldsymbol{a}, \boldsymbol{b}$. $\boldsymbol{d}$, Illustration of the placement of the ROIs for the PVS surrounding the MCA and the cortical compartment proximal to the MCA. $e$, We fitted a one-tissue compartment model to the PVS (input) and CTX (tissue) compartments, not including a $V_{\text {CSF }}$ parameter, since the tissue compartment was manually delineated to not include CSF. $\boldsymbol{f}$, Example fit of the one-tissue compartment model to the TCCs from a 7-week-old WKY. The calculated local glymphatic influx rate $\left(K_{1}\right)$ was significantly lower in the SHR than in WKY in the adult rats $(\boldsymbol{g})$, restating the results from the global one-tissue compartment analysis, although no difference was found in $k_{2}(\boldsymbol{h})$. Average modeled time-activity curves and residuals from the young (i) and adult (j) age groups. ${ }^{*} p<0.05$. RRhV, Rostral rhinal vein; CRhV, caudal rhinal vein. 
Table 5. Summary of results from the local glymphatic transport analysis

\begin{tabular}{lllll}
\hline Age (wk) & Strain & $\begin{array}{l}\text { Local } K_{1} \\
\left(\mathrm{ml} \cdot \mathrm{cm}^{-3} \cdot \mathrm{min}^{-1}\right)\end{array}$ & Local $k_{2}\left(\mathrm{~min}^{-1}\right)$ & Fit RMSE $(\%)$ \\
\hline $7-9$ & WKY & $0.047 \pm 0.015$ & $0.081 \pm 0.025$ & $11.6 \pm 4.62$ \\
& SHR & $0.036 \pm 0.010$ & $0.077 \pm 0.016$ & $11.7 \pm 3.88$ \\
$19-21$ & WKY & $0.050 \pm 0.019$ & $0.078 \pm 0.040$ & $13.4 \pm 5.77$ \\
& SHR & $0.035 \pm 0.011^{* a}$ & $0.064 \pm 0.017$ & $9.22 \pm 3.26$
\end{tabular}

${ }^{*} p<0.05$; one-tailed Mann-Whitney $U$ tests (SHR $<$ WKY); ${ }^{a} p=0.02$.

\section{Tracer uptake in corpus callosum}

Tracer uptake in corpus callosum was very low, with Gd-DOTA concentrations peaking at $\sim 0.005 \mu \mathrm{mol} / \mathrm{cm}^{3}$ in both young SHR and WKY. TCCs in corpus callosum did not differ between the 3 young SHR and 5 young WKY rats in our analysis (data not shown), and low SNR due to low uptake prevented further analysis.

\section{Gd-DOTA transport in hippocampus}

It was recently demonstrated that fluorescent tracers passively released from a needle in hippocampus spread at a higher rate in SHR than WKY with the conclusion that SHR displays enhanced drainage (Bedussi et al., 2017). To test this, we measured TACs, TTP, and AUC in the hippocampal areas. We found no significant differences in AUC (yWKY, $3.84 \pm 1.28$; ySHR, $3.39 \pm 0.76$; $p=0.52$; aWKY, $2.89 \pm 1.43$; aSHR, $2.31 \pm 1.06$; $p=0.53$; two-tailed Mann-Whitney $U$ test) or TTP (yWKY, $82.8 \pm 20.6$ min; ySHR, $89.1 \pm 20.8 \mathrm{~min} ; p=0.54$; aWKY, $94.3 \pm 14.0 \mathrm{~min}$; aSHR, $111.1 \pm 23.5 \mathrm{~min} ; p=0.26$; two-tailed Mann-Whitney $U$ test), and the direction of all nonsignificant mean differences was in the direction of decreased glymphatic flow in hippocampus.

\section{GFAP and AQP4 distribution}

To evaluate the effect of astrogliosis and AQP4 expression, histological sections from 3 young SHR, 3 young WKY, 3 adult SHR, and 3 adult WKY were immunolabeled for GFAP and AQP4 (Fig. 6). Representative micrographs of the GFAP staining (Fig. 6a-d) show the distributions of astrocytes in the cortical parenchyma around the area overlaid by the MCA. We quantified GFAP and AQP4 distribution as the percentage area covered and used the average of two micrographs per animal. Two-sample $t$ tests were applied to estimate differences, as the Mann-Whitney test is underpowered for such small sample sizes. In young animals, a significant difference was observed between WKY and SHR rats (Fig. $6 e ; 95.47 \pm 3.90$ and $75.31 \pm 2.35$, respectively, $p=0.0016$, $t=7.66, \mathrm{df}=4$; two-tailed $t$ test). Such difference was not observed in adult rats $(91.40 \pm 1.59$ and $90.54 \pm 3.12 ; p=0.69, t=$ 0.42 , df $=4$; two-tailed $t$ test). Differently from GFAP, no significant difference was observed in the immunoreactivity of AQP4 (representative micrographs are shown in Fig. $6 f-i$ ) between WKY and SHR in young or adult animals (Fig. 6j; yWKY, $55.54 \pm$ 3.14; ySHR, $59.11 \pm 0.73 ; p=0.13, t=-1.9$, df $=4$; two-tailed $t$ test; aWKY, $87.58 \pm 5.70$; aSHR, $89.74 \pm 1.52 ; p=0.56, t=$ $-0.63, \mathrm{df}=4$; two-tailed $t$ test).

\section{Discussion}

We used DCE-MRI to compare CSF flow characteristics and CSF-ISF transport kinetics as measures of glymphatic transport in young and adult SHR rats and age-matched normotensive controls (WKY) after infusing Gd-DOTA into the CM. Notably, the ventricular system anatomy and associated CSF transport pattern differed dramatically between SHR and WKY rats due to hydrocephalus in SHRs. Young and adult SHR rats displayed a 5- to 6-fold larger LV volume compared with WKY rats, along with enlargement of CM volume and $\sim 10 \%$ loss of brain volume consistent with prior reports (Ritter and Dinh, 1986; Martínez-Peña y Valenzuela et al., 2006). Despite this, CSF volume was lower in young SHR and similar in adult SHR compared with WKY. We also noted enlargement of brain ventricles with increasing age in SHR rats, also consistent with prior reports (Ritter and Dinh, 1986). Finally, we observed CSF flow dynamics with ventricular reflux in the hydrocephalic SHR rats, as has been observed in humans with hydrocephalus (Ringstad et al., 2017, 2018), which also demonstrated reflux of contrast agent into the LVs. Ventricular reflux of CSF and increased CSF backflow through the aqueduct are well-established clinical phenomena in hydrocephalus (Vanneste, 2000; Qvarlander et al., 2017).

\section{Glymphatic transport is impaired in SHR compared with WKY rats}

We developed a novel analysis method, in which we applied a one-tissue compartment model to estimate the influx rate of GdDOTA from CSF to the brain as well as the efflux rate from the brain. Our kinetic analysis demonstrated decreases in both glymphatic influx and efflux. Reduced glymphatic influx has previously been associated with impaired glymphatic system function, and thus a reduced ability of the brain to be cleared of macromolecules and fluids (Iliff et al., 2012, 2013a; Kress et al., 2014; Plog et al., 2015; Peng et al., 2016). The analyses showed lower glymphatic influx rates in SHR versus WKY rats, in both age groups, as well as reduced efflux rates in the adult SHR. A second analysis of local PVS-to-cortex transport showed a decreased glymphatic influx in adult SHR, whereas the difference of influx in young $\mathrm{SHR}$ did not reach significance. We found no differences in tracer dynamics in subarachnoid CSF surrounding CoW, implying that a comparable amount of tracer is available in the subarachnoid space at the basal brain. Thus, we conclude that the differences observed in influx parameters most likely are not caused by changes in gross flow patterns caused by ventriculomegaly in the SHR, although the study cannot provide a direct causal link between hypertension-induced vascular pathologies and reduced glymphatic influx.

Whereas previous studies have used amount of brain tracer uptake and area coverage as markers of glymphatic influx (Iliff et al., 2012, 2013a; Kress et al., 2014; Plog et al., 2015; Peng et al., 2016), the present study shows that brain tracer concentrations after CM tracer infusion depend on the efficacy of the glymphatic system, the availability of tracer in CSF proximal to brain, and the period between infusion and measurement of tracer concentration. Quantifying glymphatic influx exclusively using single-time point histological sections disregards the availability and spatiotemporal dynamics of tracer, and the current study thus highlights the importance of considering tracer distribution in CSF in glymphatic system dynamics analysis.

Bedussi et al. (2017) recently showed an increased spread of fluorescent tracer in hippocampus of aged SHR compared with WKY when passively released from the needle in hippocampus, concluding that SHR displays enhanced drainage. In apparent contradiction, we found no difference in tracer uptake in hippocampus with an insignificant tendency toward lower uptake in SHR. The experiment by Bedussi et al. (2017) was conducted in rats under 3\% isoflurane anesthesia, a concentration at which blood pressure (Bencze et al., 2013; Kato et al., 2016) and cardiac output (Seyde et al., 1987) are significantly reduced and cerebral blood flow increased in SHR versus WKY (Leoni et al., 2011). The anesthetic regimen used may have produced differing alterations 
Young
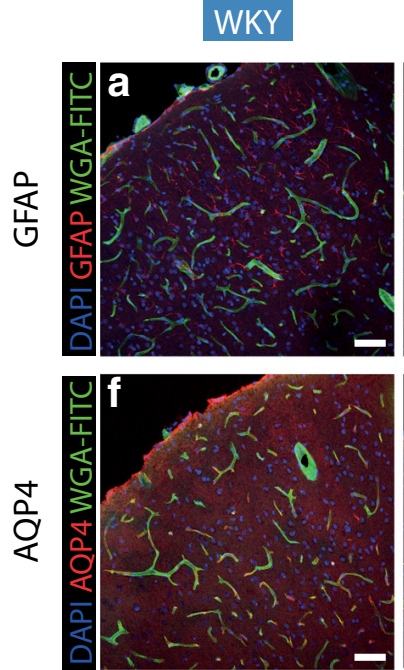
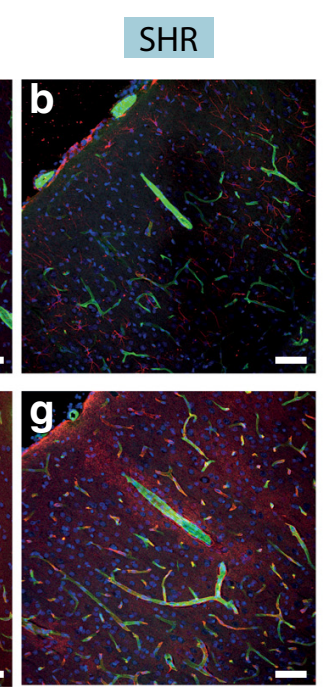

Adult

WKY
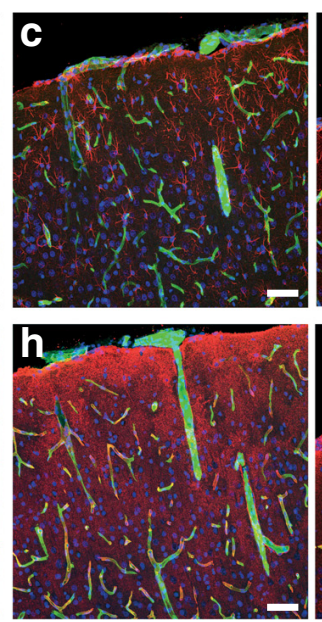
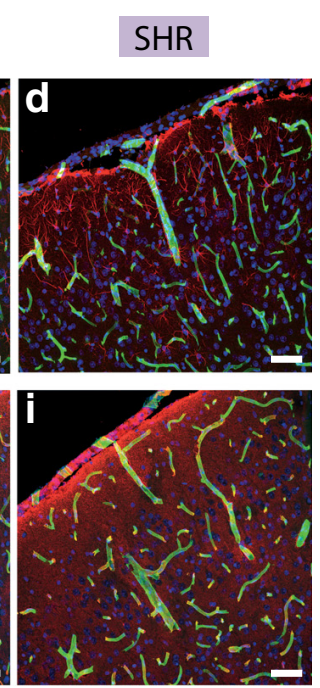

e

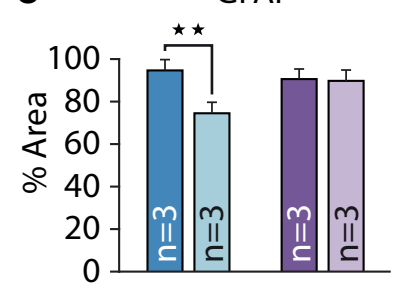

j

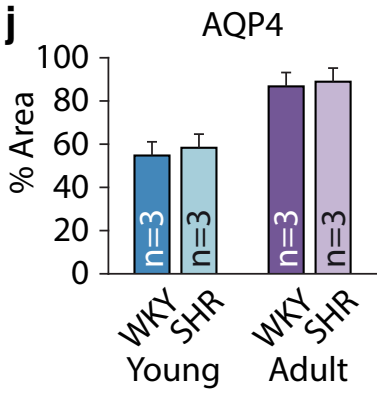

Figure 6. $\quad \boldsymbol{a}, \boldsymbol{b}$, Representative confocal micrographs from brain sections obtained from young WKY and SHR, respectively, reveal the distributions of astrocytes in the cortical parenchyma around the area overlaid by the MCA. c, d, Representative micrographs of astrocyte distribution in brain sections obtained from adult WKY and SHR, respectively. $\boldsymbol{e}$, Graphic representation of the fluorescence intensity, expressed by percentage of covered area, of GFAP immunoreactivity. In young animals, a significant difference was observed between WKY and SHR. Such difference was not observed in adult rats. $\boldsymbol{f}, \boldsymbol{g}$, Representative images of AQP4 distribution in the cortical parenchyma, also in the area overlaid by the MCA, of young WKY and SHR. $\boldsymbol{h}, \boldsymbol{i}$, AQP4 distribution in the adult brain of WKY and SHR. $\boldsymbol{j}$, Unlike GFAP, no significant difference was observed in the immunoreactivity of AQP4 between WKY and SHR in young or adult animals. Scale bars, $50 \mu \mathrm{m}$. ${ }^{* *} p<0.01$. Three animals in each group; average of 2 micrographs per animal.

in interstitial fluid motion in the two strains due to the glymphatic system's reliance on arterial wall pulsation, and thus the results of the current study may not be directly comparable. In addition, invasive procedures, such as inserting an injection pipette, is linked to a sharp reduction in glymphatic CSF tracer influx (Mestre et al., 2018b).

Hypertension-induced remodeling of the cerebral vasculature The cerebral vasculature is distinct from the peripheral vasculature because the majority of the cerebrovascular resistance is at the level of large arteries (Faraci and Heistad, 1990). Large-caliber vessels, for instance, the internal carotid artery and the MCAs, absorb most of the systolic pulse wave and are more susceptible to intraluminal pressure changes seen in hypertension (Hsu et al., 2017). When arterial pressure rises, arteries must constrict to maintain normal cerebral blood flow. Over time, this causes hypertrophic and eutrophic remodeling of the vascular wall, resulting in increased wall stiffness and a reduction in the vessel lumen (Iadecola and Davisson, 2008; Lindesay et al., 2016). Stiffening of the arterial wall could alter vessel wall pulsatility, reducing the efficiency of perivascular pumping, which is a key driver of CSF transport (Rennels et al., 1985; Hadaczek et al., 2006; Iliff et al., 2013b; Kiviniemi et al., 2016; Mestre et al., 2018a). Effective PVS fluid transport requires coordinated arterial wall motion and elasticity, which is significantly impaired in SHR rats due to vascular remodeling (Gaballa et al., 1992; Lichtenstein et al., 1998).

Vascular remodeling and glymphatic activity in other models of SVD

Diabetes also causes reduced vessel compliance and development of SVD with arteriolosclerosis (Lehmann et al., 1992; Pantoni, 2010). Patients with Type I or II diabetes exhibit hypertrophic remodeling, with increased medial cross-sectional area of the vessels (Rumble et al., 1997; Creager et al., 2003). A recent DCE-MRI study in a rat model of diabetes mellitus (Jiang et al., 2017) demonstrated a reduction by approximately two-thirds of the glym- phatic efflux rate in the diabetic rats compared with nondiabetic control rats. The study also demonstrated higher accumulation of tracer in the brain of the diabetic rats, which a second study (Davoodi-Bojd et al., 2019) attributed to reduced clearing of tracer in the diabetic group.

The main finding in the current study (i.e., decreased glymphatic influx rate) is comparable to previous findings in aging normotensive mice (Kress et al., 2014) and in the APP/PS1 murine model of AD (Peng et al., 2016). The convergent effects of hypertension, aging, diabetes, and $\mathrm{AD}$ with respect to glymphatic kinetics fit well with the general notion that hypertension accelerates aging of the brain vasculature and endothelial cells (Taddei et al., 1997; Barton et al., 2016), and that hypertension, like AD, is linked to cognitive impairment (Morris et al., 2001; Qiu et al., 2005).

One of the most important roles of the glymphatic system is removing interstitial soluble protein metabolites, such as $\mathrm{A} \beta$ and tau, culprits in $\mathrm{AD}$ and $\mathrm{VaD}$ (Iliff et al., 2012, 2014). The removal of $A \beta$ from the CNS has previously been shown to be dependent on the expression pattern of AQP4 channels in astrocytes, such that mice with deletion of AQP4 exhibited a highly significant reduction of glymphatic clearance of $A \beta$ (Iliff et al., 2012; Plog et al., 2015). The role of AQP4 in convective ISF transport was recently questioned by Smith et al. (2017) who failed to replicate earlier studies. However, using four separately generated mouse models of AQP4 disruption as well as one lacking perivascular AQP4 expression, five independent laboratories have confirmed the reliance of glymphatic transport on AQP4 expression (Mestre et al., 2018b). In postmortem brains of patients suffering from $\mathrm{AD}$ and CAA, total AQP4 expression was found to be increased (Moftakhar et al., 2010; Zeppenfeld et al., 2017). These studies highlight the importance of polarized expression of AQP4 on the astrocytic endfeet lining the PVS, rather than on the astrocyte cell body and perisynaptic processes (Kress et al., 2014; Xu et al., 2015; Peng et al., 2016). The loss of polarized AQP4 expression is associated with compromised local glymphatic activity (Iliff et al., 
2014; Kress et al., 2014). In SHR, earlier studies have shown that GFAP immunoreactivity starts to increase at $\sim 2-3$ months of age, concurrently with increased AQP4 expression (Sabbatini et al., 2000, 2002; Tomassoni et al., 2010). In the current study, we see no such differences in GFAP and AQP4 between strains, with the exception of decreased GFAP immunoreactivity in the young SHR group.

We suggest that reduced glymphatic clearance of $A \beta$ and tau could in part explain the link between AD and SVD. Future studies should aim to investigate whether similar changes in glymphatic activity exist in other models of hypertension and SVD, and whether antihypertensive treatment helps correct glymphatic dysfunction. If atherosclerosis and stiffening of cerebral vessels compromise brain waste clearance, therapeutics for maintaining vessel health and elasticity could be targets for minimizing $A \beta$ burden and impeding the progression of cognitive decline in $\mathrm{AD}$ and $\mathrm{VaD}$.

\section{References}

Adams MA, Bobik A, Korner PI (1989) Differential development of vascular and cardiac hypertrophy in genetic hypertension: relation to sympathetic function. Hypertension 14:191-202.

Aspelund A, Antila S, Proulx ST, Karlsen TV, Karaman S, Detmar M, Wiig H, Alitalo K (2015) A dural lymphatic vascular system that drains brain interstitial fluid and macromolecules. J Exp Med 212:991-999.

Avants BB, Tustison NJ, Song G, Cook PA, Klein A, Gee JC (2011) A reproducible evaluation of ANTs similarity metric performance in brain image registration. Neuroimage 54:2033-2044.

Barton M, Husmann M, Meyer MR (2016) Accelerated vascular aging as a paradigm for hypertensive vascular disease: prevention and therapy. Can J Cardiol 32:680-686.e84.

Baumbach GL, Heistad DD (1988) Cerebral circulation in chronic arterial hypertension. Hypertension 12:89-95.

Bedussi B, Naessens DM, de Vos J, Olde Engberink R, Wilhelmus MM, Richard E, Ten Hove M, vanBavel E, Bakker EN (2017) Enhanced interstitial fluid drainage in the hippocampus of spontaneously hypertensive rats. Sci Rep 7:744.

Bencze M, Behuliak M, Zicha J (2013) The impact of four different classes of anesthetics on the mechanisms of blood pressure regulation in normotensive and spontaneously hypertensive rats. Physiol Res 62:471-478.

Benveniste H, Lee H, Ding F, Sun Q, Al-Bizri E, Makaryus R, Probst S, Nedergaard M, Stein EA, Lu H (2017) Anesthesia with dexmedetomidine and low-dose isoflurane increases solute transport via the glymphatic pathway in rat brain when compared with high-dose isoflurane. Anesthesiology 127:976-988.

Chou N, Wu J, Bai Bingren J, Qiu A, Chuang KH (2011) Robust automatic rodent brain extraction using 3-D pulse-coupled neural networks (PCNN). IEEE Trans Image Process 20:2554-2564.

Creager MA, Lüscher TF, Cosentino F, Beckman JA (2003) Diabetes and vascular disease: pathophysiology, clinical consequences, and medical therapy: part I. Circulation 108:1527-1532.

Davoodi-Bojd E, Ding G, Zhang L, Li Q, Li L, Chopp M, Zhang Z, Jiang Q (2019) Modeling glymphatic system of the brain using MRI. Neuroimage 188:616-627.

Doggrell SA, Brown L (1998) Rat models of hypertension, cardiac hypertrophy and failure. Cardiovasc Res 39:89-105.

Faraci FM, Heistad DD (1990) Regulation of large cerebral arteries and cerebral microvascular pressure. Circ Res 66:8-17.

Gaballa MA, Raya TE, Simon BR, Goldman S (1992) Arterial mechanics in spontaneously hypertensive rats: mechanical properties, hydraulic conductivity, and two-phase (solid/fluid) finite element models. Circ Res 71:145-158.

Ghali MG (2017) Microsurgical technique for femoral vascular access in the rat. MethodsX 4:498-507.

Hachinski V (2015) Stroke and potentially preventable dementias proclamation: updated world stroke day proclamation. Stroke 46:3039-3040.

Hadaczek P, Yamashita Y, Mirek H, Tamas L, Bohn MC, Noble C, Park JW, Bankiewicz K (2006) The "perivascular pump" driven by arterial pulsation is a powerful mechanism for the distribution of therapeutic molecules within the brain. Mol Ther 14:69-78.
Holland PR, Searcy JL, Salvadores N, Scullion G, Chen G, Lawson G, Scott F, Bastin ME, Ihara M, Kalaria R, Wood ER, Smith C, Wardlaw JM, Horsburgh K (2015) Gliovascular disruption and cognitive deficits in a mouse model with features of small vessel disease. J Cereb Blood Flow Metab 35:1005-1014.

Hsu CY, Schneller B, Alaraj A, Flannery M, Zhou XJ, Linninger A (2017) Automatic recognition of subject-specific cerebrovascular trees. Magn Reson Med 77:398-410.

Iadecola C, Davisson RL (2008) Hypertension and cerebrovascular dysfunction. Cell Metab 7:476-484.

Iliff JJ, Wang M, Liao Y, Plogg BA, Peng W, Gundersen GA, Benveniste H, Vates GE, Deane R, Goldman SA, Nagelhus EA, Nedergaard M (2012) A paravascular pathway facilitates CSF flow through the brain parenchyma and the clearance of interstitial solutes, including amyloid beta. Sci Transl Med 4:147ral11.

Iliff JJ, Lee H, Yu M, Feng T, Logan J, Nedergaard M, Benveniste H (2013a) Brain-wide pathway for waste clearance captured by contrast-enhanced MRI. J Clin Invest 123:1299-1309.

Iliff JJ, Wang M, Zeppenfeld DM, Venkataraman A, Plog BA, Liao Y, Deane R, Nedergaard M (2013b) Cerebral arterial pulsation drives paravascular CSF-interstitial fluid exchange in the murine brain. J Neurosci 33:1819018199.

Iliff JJ, Chen MJ, Plog BA, Zeppenfeld DM, Soltero M, Yang L, Singh I, Deane R, Nedergaard M (2014) Impairment of glymphatic pathway function promotes tau pathology after traumatic brain injury. J Neurosci 34: $16180-16193$.

Jiang Q, Zhang L, Ding G, Davoodi-Bojd E, Li Q, Li L, Sadry N, Nedergaard M, Chopp M, Zhang Z (2017) Impairment of the glymphatic system after diabetes. J Cereb Blood Flow Metab 37:1326-1337.

Kaiser D, Weise G, Moller K, Scheibe J, Posel C, Baasch S, Gawlitza M, Lobsien D, Diederich K, Minnerup J, Kranz A, Boltze J, Wagner DC (2014) Spontaneous white matter damage, cognitive decline and neuroinflammation in middle-aged hypertensive rats: an animal model of early-stage cerebral small vessel disease. Acta Neuropathol Commun 2:169.

Kato K, Wakai J, Ozawa K, Sekiguchi M, Katahira K (2016) Different sensitivity to the suppressive effects of isoflurane anesthesia on cardiorespiratory function in SHR/Izm, WKY/Izm, and Crl:CD (SD) rats. Exp Anim 65:393-402.

Kim DS, Choi JU, Huh R, Yun PH, Kim DI (1999) Quantitative assessment of cerebrospinal fluid hydrodynamics using a phase-contrast cine MR image in hydrocephalus. Childs Nerv Syst 15:461-467.

Kiviniemi V, Wang X, Korhonen V, Keinänen T, Tuovinen T, Autio J, LeVan P, Keilholz S, Zang YF, Hennig J, Nedergaard M (2016) Ultra-fast magnetic resonance encephalography of physiological brain activity: glymphatic pulsation mechanisms? J Cereb Blood Flow Metab 36:1033-1045.

Kress BT, Iliff JJ, Xia M, Wang M, Wei HS, Zeppenfeld D, Xie L, Kang H, Xu Q, Liew JA, Plog BA, Ding F, Deane R, Nedergaard M (2014) Impairment of paravascular clearance pathways in the aging brain. Ann Neurol 76:845-861.

Lee H, Xie L, Yu M, Kang H, Feng T, Deane R, Logan J, Nedergaard M, Benveniste H (2015) The effect of body posture on brain glymphatic transport. J Neurosci 35:11034-11044.

Lee H, Mortensen K, Sanggaard S, Koch P, Brunner H, Quistorff B, Nedergaard M, Benveniste H (2018) Quantitative Gd-DOTA uptake from cerebrospinal fluid into rat brain using 3D VFA-SPGR at 9.4T. Magn Reson Med 79:1568-1578.

Lehmann ED, Gosling RG, Sönksen PH (1992) Arterial wall compliance in diabetes. Diabet Med 9:114-119.

Leoni RF, Paiva FF, Henning EC, Nascimento GC, Tannús A, de Araujo DB, Silva AC (2011) Magnetic resonance imaging quantification of regional cerebral blood flow and cerebrovascular reactivity to carbon dioxide in normotensive and hypertensive rats. Neuroimage 58:75-81.

Lichtenstein O, Safar ME, Mathieu E, Poitevin P, Levy BI (1998) Static and dynamic mechanical properties of the carotid artery from normotensive and hypertensive rats. Hypertension 32:346-350.

Lindesay G, Ragonnet C, Chimenti S, Villeneuve N, Vayssettes-Courchay C (2016) Age and hypertension strongly induce aortic stiffening in rats at basal and matched blood pressure levels. Physiol Rep 4:e12805.

Louveau A, Smirnov I, Keyes TJ, Eccles JD, Rouhani SJ, Peske JD, Derecki NC, Castle D, Mandell JW, Lee KS, Harris TH, Kipnis J (2015) Structural and functional features of central nervous system lymphatic vessels. Nature 523:337-341. 
Martínez-Peña y Valenzuela I, Carmona-Calero EM, Pérez-González H, Ormazabal-Ramos C, Fernández-Rodríguez P, González-Marrero I, Castañeyra-Perdomo A, Ferres-Torres R (2006) Alterations of the cerebrospinal fluid proteins and subcommissural organ secretion in the arterial hypertension and ventricular dilatation: a study in SHR rats. Histol Histopathol 21:179-185.

Mathieu E, Gupta N, Ahari A, Zhou X, Hanna J, Yücel YH (2017) Evidence for cerebrospinal fluid entry into the optic nerve via a glymphatic pathway. Invest Ophthalmol Vis Sci 58:4784-4791.

Mestre H, Tithof J, Du T, Song W, Peng W, Sweeney AM, Olveda G, Thomas JH, Nedergaard M, Kelley DH (2018a) Flow of cerebrospinal fluid is driven by arterial pulsations and is reduced in hypertension. Nat Commun 9:4878.

Mestre H, Hablitz LM, Xavier AL, Feng W, Zou W, Pu T, Monai H, Murlidharan G, Castellanos Rivera RM, Simon MJ, Pike MM, Plá V, Du T, Kress BT, Wang X, Plog BA, Thrane AS, Lundgaard I, Abe Y, Yasui M, et al. (2018b) Aquaporin-4-dependent glymphatic solute transport in the rodent brain. Elife 7:e40070.

Mitchell GF (2014) Arterial stiffness and hypertension: chicken or egg? Hypertension 64:210-214.

Moftakhar P, Lynch MD, Pomakian JL, Vinters HV (2010) Aquaporin expression in the brains of patients with or without cerebral amyloid angiopathy. J Neuropathol Exp Neurol 69:1201-1209.

Morris JC, Storandt M, Miller JP, McKeel DW, Price JL, Rubin EH, Berg L (2001) Mild cognitive impairment represents early-stage Alzheimer disease. Arch Neurol 58:397-405.

Okamoto K, Aoki K (1963) Development of a strain of spontaneously hypertensive rats. Jpn Circ J 27:282-293.

O’Rourke MF, Safar ME, Dzau V (2010) The cardiovascular continuum extended: aging effects on the aorta and microvasculature. Vasc Med $15: 461-468$

Pantoni L (2010) Cerebral small vessel disease: from pathogenesis and clinical characteristics to therapeutic challenges. Lancet Neurol 9:689-701.

Papp EA, Leergaard TB, Calabrese E, Johnson GA, Bjaalie JG (2014) Waxholm space atlas of the Sprague Dawley rat brain. Neuroimage 97: 374-386.

Phan JA, Landau AM, Jakobsen S, Wong DF Gjedde A (2017) Radioligand binding analysis of alpha 2 adrenoceptors with [(11)Clyohimbine in brain in vivo: Extended Inhibition Plot correction for plasma protein binding. Sci Rep 7:15979.

Peng W, Achariyar TM, Li B, Liao Y, Mestre H, Hitomi E, Regan S, Kasper T, Peng S, Ding F, Benveniste H, Nedergaard M, Deane R (2016) Suppression of glymphatic fluid transport in a mouse model of Alzheimer's disease. Neurobiol Dis 93:215-225.

Plog BA, Dashnaw ML, Hitomi E, Peng W, Liao Y, Lou N, Deane R, Nedergaard M (2015) Biomarkers of traumatic injury are transported from brain to blood via the glymphatic system. J Neurosci 35:518-526.

Qiu C, Winblad B, Fratiglioni L (2005) The age-dependent relation of blood pressure to cognitive function and dementia. Lancet Neurol 4:487-499.

Qvarlander S, Ambarki K, Wåhlin A, Jacobsson J, Birgander R, Malm J, Eklund A (2017) Cerebrospinal fluid and blood flow patterns in idiopathic normal pressure hydrocephalus. Acta Neurol Scand 135:576-584.
Rennels ML, Gregory TF, Blaumanis OR, Fujimoto K, Grady PA (1985) Evidence for a 'paravascular' fluid circulation in the mammalian central nervous system, provided by the rapid distribution of tracer protein throughout the brain from the subarachnoid space. Brain Res 326:47-63.

Ringstad G, Vatnehol SA, Eide PK (2017) Glymphatic MRI in idiopathic normal pressure hydrocephalus. Brain 140:2691-2705.

Ringstad G, Valnes LM, Dale AM, Pripp AH, Vatnehol SS, Emblem KE, Mardal KA, Eide PK (2018) Brain-wide glymphatic enhancement and clearance in humans assessed with MRI. JCI Insight 3:121537.

Ritter S, Dinh TT (1986) Progressive postnatal dilation of brain ventricles in spontaneously hypertensive rats. Brain Res 370:327-332.

Rumble JR, Cooper ME, Soulis T, Cox A, Wu L, Youssef S, Jasik M, Jerums G, Gilbert RE (1997) Vascular hypertrophy in experimental diabetes. role of advanced glycation end products. J Clin Invest 99:1016-1027.

Sabbatini M, Strocchi P, Vitaioli L, Amenta F (2000) The hippocampus in spontaneously hypertensive rats: a quantitative microanatomical study. Neuroscience 100:251-258.

Sabbatini M, Catalani A, Consoli C, Marletta N, Tomassoni D, Avola R (2002) The hippocampus in spontaneously hypertensive rats: an animal model of vascular dementia? Mech Ageing Dev 123:547-559.

Seyde WC, Durieux ME, Longnecker DE (1987) The hemodynamic response to isoflurane is altered in genetically hypertensive (SHR), as compared with normotensive (WKY), rats. Anesthesiology 66:798-804.

Smith AJ, Yao X, Dix JA, Jin BJ, Verkman AS (2017) Test of the 'glymphatic' hypothesis demonstrates diffusive and aquaporin-4-independent solute transport in rodent brain parenchyma. Elife 6:e27679.

Stollberger R, Wach P (1996) Imaging of the active B1 field in vivo. Magn Reson Med 35:246-251.

Taddei S, Virdis A, Mattei P, Ghiadoni L, Fasolo CB, Sudano I, Salvetti A (1997) Hypertension causes premature aging of endothelial function in humans. Hypertension 29:736-743.

Tajima A, Hans FJ, Livingstone D, Wei L, Finnegan W, DeMaro J, Fenstermacher J (1993) Smaller local brain volumes and cerebral atrophy in spontaneously hypertensive rats. Hypertension 21:105-111.

Tomassoni D, Bramanti V, Amenta F (2010) Expression of aquaporins 1 and 4 in the brain of spontaneously hypertensive rats. Brain Res 1325:155-163.

Tustison NJ, Avants BB, Cook PA, Zheng Y, Egan A, Yushkevich PA, Gee JC (2010) N4ITK: improved N3 bias correction. IEEE Trans Med Imaging 29:1310-1320.

Vanneste JA (2000) Diagnosis and management of normal-pressure hydrocephalus. J Neurol 247:5-14.

Wardlaw JM, Smith C, Dichgans M (2013) Mechanisms of sporadic cerebral small vessel disease: insights from neuroimaging. Lancet Neurol 12: 483-497.

Xu Z, Xiao N, Chen Y, Huang H, Marshall C, Gao J, Cai Z, Wu T, Hu G, Xiao M (2015) Deletion of aquaporin-4 in APP/PS1 mice exacerbates brain abeta accumulation and memory deficits. Mol Neurodegener 10:58.

Zeppenfeld DM, Simon M, Haswell JD, D'Abreo D, Murchison C, Quinn JF, Grafe MR, Woltjer RL, Kaye J, Iliff JJ (2017) Association of perivascular localization of aquaporin-4 with cognition and Alzheimer disease in aging brains. JAMA Neurol 74:91-99. 